\title{
Microsecond relaxation processes in shear and extensional flows of weakly elastic polymer solutions
}

\author{
Damien C. Vadillo ${ }^{1}$, WouterMathues ${ }^{2}$, Christian Clasen $^{2}$ \\ ${ }^{1}$ Department of Chemical Engineering and Biotechnology, University of Cambridge, \\ Cambridge. CB2 3RA, UK* \\ ${ }^{2}$ Department of Chemical Engineering, University of Leuven (KU Leuven), Willem de \\ Croylaan 46, 3001 Heverlee, Belgium \\ ${ }^{*}$ DV current address. Inca Digital Printers Ltd, Cambridge, Cambridge. CB1 3JS
}

\begin{abstract}
In this paper we introduce an experimental protocol to reliably determine extensional relaxation times from capillary thinning experiments of weakly-elastic dilute polymer solutions. The reported relaxation times for polystyrene in diethyl phthalate solutions as low as $80 \mu \mathrm{s}$ are the lowest so far assessed relaxation times in uniaxial extensional flows. This data is compared to the linear viscoelastic relaxation times that are obtained from fitting the Zimm spectrum to high frequency oscillatory squeeze flow data measured with a piezo-axial vibrator (PAV). As a result the often made statement that the extensional relaxation time reduced by the Zimm time,$\lambda_{\text {ext }} / \lambda_{z}$, is solely a function of the reduced concentration $c / c^{*}$ is not valid, but an additional dependence on the molecular weight is observed.
\end{abstract}




\section{Introduction}

Stretching a liquid drop into a filament and following the thinning dynamics enables probing the resistance of a fluid to an uniaxial stretching deformation. This fundamental material property is referred to as the extensional viscosity $\eta_{\text {ext }}$ which is, for a Newtonian liquid, constant and related to the shear viscosity $\eta_{0}$ via the Trouton ratio $\eta_{\text {ext }} / \eta_{0}=3$. The addition of a dissolved polymer to the liquid, however, increases $\eta_{\text {ext }}$ and introduces a relaxation spectrum; reviews by McKinley (2005), McKinley and Sridhar (2002) and Bach et al. (2003) provide detailed information on both experiments and modelling for a range of such viscoelastic polymer solution systems in extensional flows.

In comparison to the shear flow of polymer solutions, the experimental investigation of the viscoelastic properties in extensional flows has proven to be experimentally much more challenging [Nguyen and Kausch (1999)]. For purely uniaxial extensional flows over the last two decades the filament stretching and filament thinning methods have been developed [Sridhar (1990)]. One of the advantages of these experimental techniques is that they are viable also for lower viscosity fluids. Both filament stretching and filament thinning experiment stretch a fluid sample placed between two circular pistons into a filament and follow the thinning dynamics. A filament stretching device moves at least one of the pistons in a controlled manner so that the extension rate experienced by the fluid filament can be controlled while the stress response of the fluid to the stretching deformation is determined via a force transducer. In such configuration, the piston velocity is often non-linearly increased to ensure a constant stretch rate at the mid-filament [see for example Spiegelberg et al. (1996); Spiegelberg and McKinley (1996); Gupta et al. (2000); Anna et al. (2001)]. The three main limitations that are associated with this technique are (i) the maximum stretch rate that can be imposed to the filament (limited by the accessible piston velocities), (ii) the maximum achievable filament stretching Hencky strain (limited by the stretching distance) 
[Anna et al. (2001)], and (iii) the sensitivity of the force transducer. As a result, this technique is particularly adapted for higher viscosity polymer solutions or melts ( $>1 \mathrm{~Pa} \mathrm{~s})$.

In contrast to this, a filament thinning experiment creates an unstable filament of fluid by imposing an initial sudden step-stretch deformation to the fluid and then follows the evolution of the mid-filament diameter $D_{\text {mid }}(t)$. The thinning of the filament is controlled by a balance between inertial, viscous, elastic, gravitational and capillary forces and transient viscoelastic properties can be extracted from the observed rate of decay of $D_{\text {mid }}(t)$, as reviewed by McKinley (2005). In a filament thinning experiment, the extension rate cannot be controlled since it is only the (constant) surface tension that drives the thinning. However, as shown in the seminal work of Entov and Hinch (1997), this type of experiment enables the precise determination of the longest relaxation time of a relaxation spectrum of a viscoelastic solution in an extensional flow. In this case, the thinning dynamics of viscoelastic solution acquire an elasto-capillary balance in which the extension rate is constant and determined by this longest relaxation time of the spectrum.

Filament thinning experiments can also be performed on lower viscosity polymer solutions. The main limitation lies in the fact that the elastic stresses caused by the unraveling of the polymer chain require some deformation of the fluid before they have grown large enough to overcome solvent stresses and deliver sufficient elastic resistance to establish an elastocapillary balance [Campo-Deaño and Clasen (2010)]. For low concentrations, the required polymer deformations can be large and a visible effect of the polymer on the thinning dynamics (in comparison to the pure solvent) may occur when the finite extensibility limit is already approached. Nonetheless, filament thinning experiments have been proven to be very useful to interrogate the extensional properties of low viscosity and/or low viscoelasticity solutions [Plog et al. (2005); Rodd et al. (2005); Yesilata et al. (2006); Sattler et al. (2008); Vadillo et al. (2010a); Campo-Deaño and Clasen (2010)] and for small fluid volumes [Kojic 
et al. (2006); Erni et al. (2011)]. Rodd et al. (2005), for example, realized a series of experiments on aqueous PEO solutions and established a lower limit of viscosity of $70 \mathrm{mPa} . \mathrm{s}$ for a purely Newtonian fluid and lowest relaxation time $\lambda \sim 1 \mathrm{~ms}$ for a viscoelastic fluid when using a commercially available capillary break up extensional rheometer (CaBER). Another limitation of the filament thinning method for the investigation of lower viscosity liquids is the initial separation velocity of the pistons. In order to observe the filament thinning the time $\delta t_{0}$ to achieve a given stretching distance must be shorter than the break up time $t_{\mathrm{bu}}$ of the filament. Recently, Vadillo et al. (2010) introduced with the "Cambridge Trimaster" (CTM) a fast capillary thinning extensional rheometer that can probe break-up time as short as $5 \mathrm{~ms}$ and therefore fluids with viscosities down to $10 \mathrm{mPa}$ s and relaxation times as short as $\sim 200$ $\mu$ s. However, the authors also indicated that, similar to the observations of Rodd et al. [Rodd et al. (2005)], at the necessary high stretching velocities and large strain rates the inertia of the fluid cannot be neglected and leads to oscillations of the filament. Recently, Campo-Deaño and Clasen (2010) introduced the slow retraction method (SRM) as an alternative way to investigate filament thinning mechanism of fluids with viscosities of order of water and very short relaxation times. This technique consists in slowly separating the pistons close to the critical separation distance at which a statically stable liquid bridge still exists. Subsequently the filament is very slowly further extended until the filament becomes unstable and the thinning and breaking process is initiated. This avoids inertia effects due to fast stretching motions and pistons oscillations. Using this alternative technique, the authors have been able to extract relaxation as short as $200 \mu$ s for dilute aqueous solutions of poly(ethylene oxide) with a molecular weight of $10^{6} \mathrm{~g} / \mathrm{mol}$ and viscosities between 1 and $3 \mathrm{mPa}$ s. Recent experiments by Sharma et al. (2010) on the breakup of weakly viscoelastic jets of dilute aqueous solutions of poly(ethylene oxide) with a molecular weight of $3 \times 10^{5} \mathrm{~g} / \mathrm{mol}$, viscosity 
of $3.7 \mathrm{mPa}$ s demonstrated that such jetting flows can also be used to extract extensional relaxation times as short as $170 \mu \mathrm{s}$.

Still, many industrial processes involve fluids with low viscosities and shorter polymer molecules leading to even shorter relaxation times then the ones reported above. The electro spinning process for example involves sub-millisecond relaxation processes of polymer solutions [Regev et al. 2010]. Also inkjet printing, for example, comprises fluid viscosities between 3 and $20 \mathrm{mPa}$ s and low molecular weight polymer additive are used to stabilize the droplet ligature and prevent satellite formation [Hoath et al. (2009)]. A downside of the addition of polymers is the increase of the extensional viscosity of the fluid and subsequently the significant reduction of jetting speed and increase of the length of the droplet filament. An optimization of the polymer addition to balance an extensional viscosity increase and the satellite formation requires techniques that allow determining relaxation time even on the microsecond level.

The linear viscoelastic properties of fluids with such short relaxation times can be assessed with high frequency rheology and it has been demonstrated that dilute polymer solutions can exhibit linear viscoelastic relaxations time that are comparable with jetting processes $(\sim 30 \mu \mathrm{s})$ [Vadillo et al. (2010b)]. However, filament thinning processes are generally highly non-linear and the addition of low amounts of polymer leads to relaxation spectra in extensional deformation that are different from linear deformations. For very dilute solutions Clasen et al. (2006a) demonstrated that, for high molecular weight polystyrene, the extensional relaxation time is of same order of magnitude as the shear relaxation time and comparable to the longest Zimm relaxation time. However, for concentrations just below the critical coil overlap concentration $c^{*}$, the relaxation times in extension have been reported to be orders of magnitude larger than shear relaxation times [Entov and Yarin (1984); Basilevsky et al. (2001); Stelter et al. (2002); Tirtaatmadja et al. (2006); Clasen et al. (2006a)]. For dilute 
solutions of lower molecular weight polymers this deviation of relaxation times in shear and extension is also expected, but so far not investigated due to the discussed experimental limitations of determining very short relaxation times in extensional flows.

The present work is therefore focusing on the determination of the very short extensional relaxation times of dilute, low molecular weight polymer solutions using the CTM. First the experimental requirements to investigate the thinning dynamics of weakly elastic dilute polymer fluids and to reliably determine relaxation times on the microsecond level are presented. Secondly, we are comparing them to the linear viscoelastic relaxation times obtained from high frequency PAV measurements, focussing in the third part on dilute polymer solutions with similar overlap concentrations $c / c^{*}$ but different molecular weights.

\section{Materials and methods}

\subsection{Linear viscoelastic measurements}

The low viscosity fluids investigated here exhibit relaxation times of milliseconds or below. Regular rotational rheometry can therefore not be used to characterize their relaxation behaviour, in particular since the applicability of time-temperature superposition is limited due to the low flow activation energy of polymer solutions [Vananroye et al. (2011)] and the sensitive nature of these fluids prevents measurements over a wide temperature range. In order to access the low relaxation times in the present work, a piezo-axial vibrator (PAV) has been used. The PAV (Fig. 1) is a dynamic squeeze flow rheometer used to characterise the linear viscoelastic (LVE) response of liquids with viscosities as low as $1 \mathrm{mPa} . \mathrm{s}$ over a range of frequencies from $0.1 \mathrm{~Hz}$ to $10000 \mathrm{~Hz}$ [Crassous et al. (2005), Hoath et al. (2009), Vadillo et al. (2010b)]. The current set up uses piezoelectric elements (PZTs) to excert a vibrational force $F$ to the cylindrical fluid sample confined $(5-200 \mu \mathrm{m})$ between the upper and lower plate

(Fig. 1). The resulting displacement of the excited lower plate with an amplitude of $\sim 5 \mathrm{~nm}$ is 
determined with a second set of PZTs and the resulting phase shift and amplitude $x_{0}$ for the unloaded and $x$ for the sample loaded PAV are determined with a lock-in amplifier (SR 850, Stanford Research Systems). The phase shift and the ratio $x / x_{0}$ enable calculating the stiffness of the fluid $K^{*}$ that can be related to the complex modulus $\mathrm{G}^{*}$ using standard squeeze flow linear viscoelastic analysis [Kirschenmann (2003)]:

$$
K^{*}=\frac{\frac{3 \pi}{2} R\left(\frac{R}{d}\right)^{3} G^{*}}{1+\frac{\rho \omega^{2} d^{2}}{10 G^{*}}+\ldots}
$$

where $\rho$ is the sample density, $\omega$ the angular frequency of oscillation and $G^{*}$ the complex modulus of the test fluid with $G^{*}=G^{\prime}+\mathrm{i} G^{\prime}$ ' where $G^{\prime}$ is the storage modulus and $G^{\prime \prime}$ is the loss modulus. The denominator in eq. (1) represents the first order series development of the inertia contribution that has to be taken into account at high frequency. This fist order

approximation is sufficient as long as $\frac{\rho \omega^{2} d^{2}}{10 G^{*}}<0.35$. The gap distances between the upper and lower plate used in the following were 25 and $35 \mu \mathrm{m}$, corresponding to a maximum strain of $0.02 \%$ with which the experiments were performed within the linear response regime for all tested fluids over the measured frequency range. A detailed description of the PAV and the signal analysis can be found elsewhere [Groß et al. (2002); Kirschenmann (2003); Crassous et al. (2005); Vadillo et al. (2010b)].

\subsection{Capillary thinning extensional rheometry}

The capillary thinning experiments were performed using the Cambridge Trimaster [Vadillo et al. (2010a)]. This apparatus rapidly stretches a cylinder of fluid initially placed between two pistons which symmetrically move in an opposite (z-) direction (Fig. 2). The symmetric movement enables the mid-filament to remain at a fixed position and therefore an easy observation of the mid-filament diameter with high-speed imaging. Filament thinning 
experiments were performed at ambient temperature by placing the sample fluid between pistons with a diameter of $D_{0}=1.2 \mathrm{~mm}$ and stretching it from an initial piston separation distance $L_{0}$ to a final distance $L_{\mathrm{f}}$. A high speed camera (Photron Fastcam 1024 PCI) was used to record the filament profile $D(z, t)$ evolution and to determine in particular the thinning of the mid-filament $D_{\text {mid }}(t)$ and the minimum filament diameter $D_{\min }(t)$. The resolution and frame rate used were $1024 \times 1024$ square pixels at 1000 frames per second, and $32 \times 32$ square pixels at $10^{5}$ frames/s with a minimum shutter time of $3 \mu \mathrm{s}$. The illumination was provided by a continuous fibre optic light source. The spatial resolution of the optical setup is of order of $5.6 \mu \mathrm{m} /$ pixel which means that the extensional rheology of the fluid can be determined up to a maximum Hencky strain of 10.7. Further details on the Cambridge Trimaster capabilities and limitations can be found in Vadillo et al. (2010a).

The obtained images were subsequently analysed with an algorithm based on the Matlab image processing toolbox in order to locate the edges of the filament and find the position and magnitude of the minimum filament diameter $D_{\min }(t)$. The edges were identified by a modified Marr-Hildreth algorithm with a Gaussian low pass filter with a standard deviation of 1.0 to achieve edge detection with sub-pixel accuracy (Marr and Hildreth (1980), Huertas and Medioni (1986)). Since the use of this small standard deviation makes the image detection rather susceptible to noise, the actual filament edges are subsequently distinguished from the background noise by a line detection algorithm using the a priori knowledge of the shape of the filament.

In order to obtain viscoelastic material functions from the diameter data of the thinning filament one has to identify which fluid property is resisting the capillary forces. For the thinning of a low viscosity fluid the main initial resistance against the capillary forces are originating from the inertia of rearranging fluid elements (rather than from the fluid viscosity). In this case of an inertia-capillary balance, the diameter decay is following a power law with a characteristic exponent of 2/3 [Brenner et al. (1996), Day et al. (1998), Rodd et al. (2005)]: 
$D_{\min }(t)=1.28\left(\frac{\sigma}{\rho}\right)^{\frac{1}{3}}\left(t_{b u}-t\right)^{\frac{2}{3}}$

where $\sigma$ is the surface tension, $\rho$ the density and $t_{\mathrm{bu}}$ the time at filament break-up. The addition of polymer results in the appearance of an extra tensile stress $\Delta \tau_{\mathrm{p}}$ that eventually slows the thinning dynamics of the inertia-capillary balance. In case that this polymeric stress $\Delta \tau_{\mathrm{p}}$ becomes large enough to balance the surface pressure in the thinning filament, the midfilament diameter has been predicted to decay exponentially [Basilevski et al. (1990); Reynardy (1994, 1995); Brenner et al. (1996); Bazilevksi et al. (1997); Eggers (1997); Entov and Hinch (1997)]. The theoretical prediction of an elasto-capillary balance has been observed experimentally for high-viscosity polymer solutions [Bazilevsky et al. (1990); Liang and Mackley (1994); Kolte et al. (1999); Anna and McKinley (2001); McKinley (2005); Ma et al. (2008); Miller et al. (2009); Clasen (2010)] as well as low viscosity polymer solutions [Rodd et al. (2005), Tuladhar and Mackley (2008), Vadillo et al. (2010a); Campo-Deaño and Clasen (2010);)]. The decay rate in the measured diameter depends only on the longest extensional relaxation time $\lambda_{\text {ext }}$ and is given for dilute polymer solutions by [Entov and Hinch (1997); Clasen et al. (2006b)]:

$D_{\text {mid }}(t)=D_{0}\left(\frac{G D_{0}}{4 \sigma}\right)^{1 / 3} \exp \left(\frac{-t}{3 \lambda_{\text {ext }}}\right)$

where $G=c R T / M_{\mathrm{w}}$ is the modulus of the dilute solution. The longest extensional relaxation time $\lambda_{\text {ext }}$ can then easily be extracted from fitting the exponential thinning regime on the diameter evolution. The conditions for a transition from an inertia-capillary to an elastocapillary balance depend on the polymer concentration and molecular weight. The discussion if it is actually possible to see this transition and to do a valid fit with eq. (3) is done in detail in the Results and Discussion section.

Independent of the choice of the dominant balancing force, it is always possible to formulate an apparent extensional viscosity $\eta_{e, \text { app }}$ from the mid filament diameter evolution by taking the ratio between the capillary pressure $2 \sigma / D_{\text {mid }}(t)$ within the filament and the strain rate 
$\dot{\varepsilon}=\frac{-2}{D_{\text {mid }}(t)} \frac{d D_{\text {mid }}(t)}{d t}$,

to give [Anna and McKinley (2001)]:

$\eta_{e, a p p}=-\frac{\sigma}{\frac{d D_{\text {mid }}(t)}{d t}}$

In order to perform the required differentiation of experimental data in eqs. (4) and (5), the filament diameter data were further processed using a Savitzky-Golay filter with a fifth order polynomial and a fitting window of 15 points. This technique has been preferred to a weighted adjacent averaging as it tends to better preserve features of the data.

\subsection{Sample preparation and characterization}

The test fluids were multiple series of monodisperse polystyrene dissolved in diethyl phthalate (DEP). Five stock solutions of monodisperse polystyrene (PS) with different molecular weight dissolved at $10 \mathrm{wt} \%$ in diethyl phthalate (DEP) were prepared by adding the PS to the DEP at ambient temperature. The resulting solution was heated up to $180^{\circ} \mathrm{C}$ and stirred for several hours until the polymer was fully dissolved. Five concentration series (referred to as series I-V) over a range of $0.2-2 \mathrm{wt} \%$ were prepared by subsequent dilution of the respective stock solution. The series I was prepared from PS with a molecular weight of $M_{\mathrm{w}}=70 \mathrm{~kg} / \mathrm{mol}$ (PS70) manufactured by Sigma-Aldrich. The four other PS samples with $M_{\mathrm{w}}$ of $110 \mathrm{~kg} / \mathrm{mol}$ (PS110), $210 \mathrm{~kg} / \mathrm{mol}$ (PS210), $306 \mathrm{~kg} / \mathrm{mol}$ (PS306) and $488 \mathrm{~kg} / \mathrm{mol}$ (PS488), were manufactured by Dow and were used to formulate dilution series II, III, IV and V respectively. A sixth solution series (VI) with different $M_{\mathrm{w}}$ and polymer concentration $c$ but the same zero-shear viscosity of $\eta_{0} \sim 12$ Pa.s was prepared for the filament thinning experiments. The four samples of series VI were PS110 at $0.5 \mathrm{wt} \%$, PS2 10 at $0.4 \mathrm{wt} \%$, PS306 at $0.2 \mathrm{wt} \%$ and PS488 at $0.1 \mathrm{wt} \%$ in DEP respectively. Mass and number average molecular weights $M_{w}$ and $M_{n}$ as well as polydispersity $M_{w} / M_{n}$ were determined using gel permeation chromatography (GPC) with THF as the solvent. All polymer and polymer solution properties are summarised in Table 1. 
The surface tension was measured using a Wilhelmy plate (NIMA DST 9005, NIMA Technology, England) and a "SITA, pro-line t15" bubble pressure tensiometer (Messtechnik $\mathrm{GmbH}$, Germany). A constant value of $37 \mathrm{mN} / \mathrm{m}$ was obtained for both the pure DEP and the PS in DEP solutions up to $5 \mathrm{wt} \%$ (PS110 in DEP).

The intrinsic viscosity $[\eta]$, the critical overlap concentration of entanglement $c^{*}$, and the radius of gyration of the carbon-carbon bonds $R_{\mathrm{g}}$ have been obtained for the different molecular weights (Fig. 3) as described in [Anna et al. (2001); Clasen et al. (2006a)]. The intrinsic viscosity $[\eta]$ has been obtained from the zero-shear viscosities $\eta_{0}$ of the different concentration series using the Huggins-Kramer extrapolation of the viscosity data to infinite dilution:

$$
\frac{\left(\frac{\eta_{0}}{\eta_{s}}-1\right)}{c}=[\eta]+k_{H}[\eta]^{2} c+\ldots
$$

where $\eta_{\mathrm{s}}$ is the solvent viscosity (with a viscosity of the pure solvent DEP of $\eta_{\mathrm{s}}=9.9 \mathrm{mPa} . \mathrm{s}$ ) and $k_{\mathrm{H}}$ is the Huggins coefficient. The viscosities $\eta_{0}$ of the solutions were determined from PAV low frequency complex viscosity $\eta^{*}$ data within the terminal relaxation regime, and for lower polymer concentrations with an Ubbelohde capillary viscometer (capillary Ic; the viscosities for the different concentration series are shown in Fig. 5a). The radius of gyration $R_{\mathrm{g}}$ was then obtained from the Flory-Fox equation:

$$
R_{g}=\left(\frac{[\eta] M_{w}}{\Phi_{0}}\right)^{\frac{1}{3}}
$$

where $M_{\mathrm{w}}$ is the molecular weight and $\Phi_{0}$ is the Flory constant equal to $3.67 .10^{24} \mathrm{~mol}^{-1}$. The critical polymer overlap concentrations $c^{*}$ have been calculated from the ideal volume requirement $4 \pi R_{g}^{3} / 3$ of a polymer coil, using the radius obtained from the experimentally determined intrinsic viscosity via the Flory Fox equation of eq. (7) [Graessley (1980); Harrison et al. (1998)]: 


$$
c^{*}=\frac{M_{w} / N_{A}}{\frac{4}{3} \pi R_{g}^{3}}=\frac{\Phi_{0}}{\frac{4}{3} \pi N_{A}[\eta]}
$$

where $N_{\mathrm{A}}$ is the Avogadro number equal to $6.0210^{23} \mathrm{~mol}^{-1}$ and therefore $3 \Phi_{0} /\left(4 \pi N_{A}\right)=$ 1.45 .

The polymer extensibility $L$, representing the ratio of a fully extended polymer (dumbbell) to its equilibrium length can be described in terms of molecular parameters as:

$L=\sqrt{3}\left[\frac{j(\sin \theta / 2)^{2} M_{w}}{C_{\infty} M_{u}}\right]^{1-v}$

where $\theta$ is $\mathrm{C}-\mathrm{C}$ bond angle, $j$ is the number of bonds of a monomer of molar mass $M_{\mathrm{u}}, C_{\infty}$ is the characteristic ratio for a given polymer-solvent system and $v$ is the excluded volume exponent. For polystyrene, the values are $\theta=109.5^{\circ}, j=2, M_{\mathrm{u}}=104 \mathrm{~g} / \mathrm{mol}$, and $C_{\infty}=9.6$ [Anna et al. (2001); Clasen et al. (2006a)]. The different polymer solution properties are summarised in Table 1.

The excluded volume exponent $v$ for PS in DEP has been obtained from the relation $[\eta] \sim M_{w}^{3 v-1}$. For this, the intrinsic viscosity data of Table 1 are completed by data provided in [Clasen et al. (2006a)] for polystyrene with higher molecular weights of 2.84, 5.67 and 8.27 $10^{6} \mathrm{~g} / \mathrm{mol}$ in DEP. Figure 3 shows the intrinsic viscosity (and radius of gyration) obtained as a function of the different molecular weights. The fit of these data to $[\eta] \sim M_{w}^{3 v-1}$ (or $R_{g} \sim M_{w}^{v}$ respectively) results in an excluded volume exponent of $v=0.555$ for polystyrene in DEP in good agreement with [Clasen et al. (2006a)]. 


\begin{tabular}{|c|c|c|c|c|c|c|c|c|c|c|}
\hline & $\begin{array}{c}M_{\mathrm{W}} \\
(\mathrm{kg} / \mathrm{mol})\end{array}$ & $\begin{array}{c}M_{\mathrm{n}} \\
(\mathrm{kg} / \mathrm{mol})\end{array}$ & $M_{\mathrm{w}} / M_{\mathrm{n}}$ & $c^{*}$ & $\begin{array}{c}R_{\mathrm{g}} \\
(\mathrm{wt} \%)\end{array}$ & $\begin{array}{c}{[\eta]} \\
(\mathrm{nm})\end{array}$ & $\begin{array}{c}L \\
(\mathrm{ml} / \mathrm{g})\end{array}$ & $\begin{array}{c}\sigma \\
(\mathrm{mN} / \mathrm{m})\end{array}$ & $\begin{array}{c}\rho \\
\left(\mathrm{kg} / \mathrm{m}^{3}\right)\end{array}$ & $\begin{array}{c}\lambda_{\mathrm{z}} \\
(\mu \mathrm{s})\end{array}$ \\
\hline PS70 & 70 & 71 & 1.056 & 6.96 & 7.24 & 25 & 12.2 & 37 & 1120 & 3.3 \\
\hline PS110 & 110 & 105 & 1.128 & 3.74 & 10.35 & 37 & 14.8 & 37 & 1120 & 7.8 \\
\hline PS210 & 210 & 181.4 & 1.146 & 2.80 & 14.15 & 49.54 & 21.3 & 37 & 1120 & 20.0 \\
\hline PS306 & 306 & 260.8 & 1.169 & 2.49 & 16.67 & 55.57 & 25.2 & 37 & 1120 & 32.9 \\
\hline PS488 & 488 & 410.15 & 1.191 & 1.53 & 22.93 & 90.7 & 31.0 & 37 & 1120 & 83.8 \\
\hline
\end{tabular}

Table 1: Characteristic parameters of the polystyrene samples dissolved in diethyl phthalate.

\section{Results and discussion}

\subsection{High frequency shear rheology}

The PAV has been used to characterize the high frequency behaviour with the aim to obtain the linear viscoelastic (LVE) relaxation times of the samples. Experimental results obtained for series II (PS110 in DEP) are used as an example of the behaviour of the different series and are presented in Fig. 4. The loss modulus $G^{\prime}$ ' and the storage modulus $G^{\prime}$ approach at lower frequencies the terminal relaxation regime with the expected scalings with a power of 1 for the loss modulus, 2 for the storage modulus, and a constant complex viscosity $\eta$ in this regime. These experimental observations are valid for all the series of fluids although the data are not explicitly reported here.

The longest relaxation time has been determined by fitting the Zimm model for dilute polymer solutions to the measured PAV data of the linear viscoelastic moduli $G^{\prime}(\omega)$ and $G^{\prime \prime}(\omega)$ :

$$
\begin{aligned}
& G^{\prime}(\omega)=G \sum_{i=1}^{N_{\text {modes }}}\left(\frac{\left(\lambda_{0} \omega\right)^{2}}{i^{4+2 \%}+\left(\lambda_{0} \omega\right)^{2}}\right) \\
& G^{\prime \prime}(\omega)=\eta_{s} \omega+G \sum_{i=1}^{N_{\text {modes }}}\left(\frac{\left(\lambda_{0} \omega\right) i^{2+\& \%}}{i^{4+2 \%}+\left(\lambda_{0} \omega\right)^{2}}\right)
\end{aligned}
$$


where $G=c R T / M_{w}$ is the modulus, $\mathrm{R}=8.314 \mathrm{~J} / \mathrm{mol} . \mathrm{K}$ is the universal gas constant, $\omega$ is the angular frequency, $\mathrm{T}$ is the absolute temperature, and $v$ is the measure of the hydrodynamic interaction between the segments of the polymer chain and the surrounding solvent. This parameter was determined to be $v=-0.335$ from the approximation $3 v=2+\widetilde{\sigma}$ [Anna et al. 2001)]. In the terminal relaxation regime eq. (10) reduces to

$$
\begin{aligned}
& \lim _{\omega \rightarrow 0} G^{\prime}(\omega)=G\left(\lambda_{0} \omega\right)^{2} \sum_{i=1}^{N_{\text {modes }}} \frac{1}{i^{4+2 \& 0}} \\
& \lim _{\omega \rightarrow 0} G^{\prime \prime}(\omega)=\eta_{s} \omega+G \lambda_{0} \omega \sum_{i=1}^{N_{\text {modes }}} \frac{1}{i^{2+\& \%}}
\end{aligned}
$$

Examples of the fits of eqs. (10) and (11) to the PAV data of the fluids of series II are given in Fig. 4, and a good agreement could be obtained when using at least eight modes. The longest relaxation times $\lambda_{0, \text { fit }}$ obtained from these fits for the five different samples and concentration series are given in Fig. $5 \mathrm{~b}$ as the reduced values $\lambda_{0, f i t} / \lambda_{z}$. The theoretical concentration independent Zimm relaxation time

$$
\lambda_{z}=\frac{1}{\sum_{i=1}^{N_{\text {modes }}} \frac{1}{i^{2+80}}} \frac{[\eta] \eta_{s} M_{w}}{R T}
$$

that is observed as the lower limit for dilute solutions is also given in Table 1. The sum $\sum_{i=1}^{N_{\text {modes }}} \frac{1}{i^{2+\phi r}}$ in eq. (12) is equivalent to $U_{n k \text {, }}$ the universal ratio of the characteristic relaxation time $\lambda_{\eta}=\left(\eta_{0}-\eta_{s}\right) / G$ of a dilute polymer system to the longest relaxation time $\lambda_{0}$, and $U_{\eta \times}=$ $\lambda_{\eta} / \lambda_{0}=2.11$ for the polymer solutions in this paper [Öttinger (1996)].

The onset of increase of the longest relaxation time of the LVE experiments $\lambda_{0}$ with concentration above the lowest limit of the Zimm relaxation time $\lambda_{z}$ can be estimated from the increase of the polymer contribution to the viscosity $\eta_{p}=\left(\eta_{0}-\eta_{s}\right)$ with the concentration. Inserting in $\lambda_{0}=U_{\eta \lambda} \eta_{p} / G$ for example the Martin equation $\eta_{p}=\eta_{s} c[\eta] \exp \left(k_{M} c[\eta]\right)$ in combination with eqs. (8) and (12) [Clasen et al. (2006a)] gives:

$$
\lambda_{0}=\lambda_{z} \exp \left(1.46 k_{M} \frac{c}{c^{*}}\right)
$$


where $k_{M}$ is the Martin coefficient [Kulicke and Clasen (2004)]. Figure 5a shows a fit of the Martin equation to the zero-shear viscosity of dilution series in this study and a $k_{M}$ of 0.37 has been obtained. This value is consistent with a $k_{M}$ of 0.35 reported previously for polystyrene of higher molecular weight in DEP [Clasen et al. (2006a)]. Equation (13) indicates that the increase of the longest relaxation $\lambda_{0} / \lambda_{z}$ will be solely a function of the overlap parameter $c / c^{*}$. Figure $5 \mathrm{~b}$ shows that, at least for the LVE measurements, this is also experimentally observed.

The same assumption (that $\lambda_{0} / \lambda_{z}$ is constant for the same overlap parameter $c / c^{*}$, independent of the molecular weight of the polymer) has also been made for the longest extensional relaxation time in several publications [e.g. Plog et al. (2005); Tirtaatmadja et al. (2006); Clasen et al. (2006a); Arnolds et al. (2010)]. In order to probe this assumption, a series of $c / c^{*}$ - matched solutions of different molecular weights (and $c / c * \sim 0.1$ ) has been prepared for different polymer molecular weights. From eqs. (6) and (8) it follows that these solutions have the same zero shear viscosity and are thus 'viscosity matched'.

The PAV data for this viscosity matched series VI are presented in Fig. 6. The similarity of the loss modulus G" for different molecular weights in the terminal relaxation regime (and therefore also of the complex viscosity) indicates that the viscosity of the samples is indeed similar for the same $c / c^{*}$. The $G^{\prime}$ data shows, as expected, differences between the samples and the resulting different longest relaxation times $\lambda_{0, f i t}$ obtained from fitting the terminal relaxation regime of $G^{\prime}$ and $G^{\prime}$ in Fig. 6 with eq. (11) are given in Table 2 (and are also included in Fig. 5b). Despite the differences in $\lambda_{0 \text {,fit }}$ from the LVE data, the reduced data $\lambda_{0, \text { fit }} / \lambda_{Z}$ in Fig $5 \mathrm{~b}$ and Table 2 are similar for the different molecular weights as expected from eq. (13). 
With this set of viscosity matched fluids it is therefore possible to probe the validity of the often made assumption that $\lambda_{0} / \lambda_{Z}$ is also constant for the same $c / c^{*}$ in an uniaxial extensional flow. This comparison is done in the following section.

\begin{tabular}{|c|c|c|c|c|c|c|c|c|}
\hline $\begin{array}{c}M_{\mathrm{w}} \\
(\mathrm{kg} / \mathrm{mol})\end{array}$ & $\begin{array}{c}c \\
(\mathrm{wt} \%)\end{array}$ & $\begin{array}{c}c_{\text {low }} \\
(\mathrm{wt} \%)\end{array}$ & $\begin{array}{c}\eta^{*} \\
(\mathrm{mPa} . \mathrm{s})\end{array}$ & $\begin{array}{c}\lambda_{0, \mathrm{fit}} \\
(\mu \mathrm{s})\end{array}$ & $\lambda_{0, \mathrm{fit}} \lambda_{\mathrm{Z}}$ & $\begin{array}{c}\lambda_{\text {ext }} \\
(\mu \mathrm{s})\end{array}$ & $\Delta \lambda_{\text {ext }}$ & $\lambda_{\text {ext }} \lambda_{\mathrm{z}}$ \\
\hline 110 & 0.5 & 0.081 & 12.4 & 11 & 1.41 & 197 & 16 & 25.2 \\
\hline 210 & 0.4 & 0.046 & 12.5 & 22 & 1.10 & 92 & 14 & 4.6 \\
\hline 306 & 0.2 & 0.041 & 11.6 & 42 & 1.28 & $(\sim 30)$ & 5 & $(\sim 0.93)$ \\
\hline 488 & 0.1 & 0.029 & 11 & 80 & 0.95 & 83 & 5 & 1.03 \\
\hline
\end{tabular}

Table 2: Rheological parameters of the fluids of series VI.

\subsection{Capillary thinning}

\section{Experimental requirements}

The visualization of filament thinning of low viscosity weak elastic fluids requires several experimental conditions to be met: (i) the filament stretching must be fast enough so that the filament does not break before the piston stops, (ii) the optical magnification must be high enough to resolve the filament diameter, (iii) the capture frame rate must be fast enough to observe an elasticity controlled exponential filament decay.

(i) The filament thinning velocity of a fluid is controlled by the competition between its surface tension, which is the driving thinning force, and viscosity, inertia, or elasticity which stabilise the filament. This competition can be summarised using three dimensionless numbers, namely the Ohnesorge number $O h$ that represents the competition between viscosity and inertia, the Deborah number De for elasticity and inertia, and the elasto-capillary number Ec that compares elasticity and viscosity [McKinley (2005), Clasen et al. (2011)],

$$
O h=\frac{\eta}{\sqrt{\rho \sigma R}}, \quad D e=\frac{\lambda}{\sqrt{\rho R^{3} / \sigma}}, \quad E c=\frac{\lambda \sigma}{\eta R},
$$


where $\sigma$ is the surface tension, $R$ is the filament radius, $\rho$ is the density, $\eta$ is the viscosity and $\lambda$ is the fluid longest relaxation time. For fluids of series VI, the low initial Ohnesorge number $O h \sim 0.06$ and (with an estimated relaxation time $\lambda$ of $\sim 100 \mu \mathrm{s}$ ) the low elasto-capillary number $E c \sim 0.06$ indicate that the fluids will behave inviscid. Furthermore, the low Deborah number $D e \sim 0.04$ ) of these inviscid fluids indicates that they will show an initially inertia controlled filament thinning [Clasen et al. (2009); Clasen et al. (2011)]. This explains the top and bottom break-up previously reported for similar solution of PS in DEP [Vadillo et al. (2010a)]. The time to break-up of such an inertia controlled thinning is given by $t_{i}=1.95 \sqrt{\frac{\rho R_{0}^{3}}{\sigma}}[$ Rodd et al. (2005)] and will be for the fluids of series VI $\sim 5 \mathrm{~ms}$. The time $\delta t_{0}=\frac{L_{f}-L_{0}}{2 V_{p}}$ required by the apparatus to perform the axial stretching distance $\left(\mathrm{L}_{\mathrm{f}}-\mathrm{L}_{0}\right)$ must then be shorter than this critical time scale for inertio-capillary break-up. For a stretching distance of $0.8 \mathrm{~mm}$, this requires a piston velocity $V_{\mathrm{p}}>150 \mathrm{~mm} / \mathrm{s}$.

(ii) The dissolved polymer will eventually cause the onset of an elasto-capillary balance in the thinning process. In order to resolve for very small filaments the expected exponential decay of the radius following eq. (3), the optical resolution of the images needs to be sufficiently high. A comparison of the stabilization effects on the filament via the Deborah number of eq. (14) indicates a transition from an inertial to an elasticity controlled thinning at a critical Deborah number of $D e \geq 1$ [Clasen et al. (2011)]. From this and with an estimated relaxation time of $\sim 100 \mu \mathrm{s}$ for the investigated critical system, one expects the transition to an exponential thinning regime to occur for filament radii $R \leq \sqrt[3]{\lambda^{2} \sigma / \rho}=70 \mu \mathrm{m}$. Balancing the spatial resolution and the field of view of the imaging system, an adequate resolution of 5 $\mu \mathrm{m} / \mathrm{pixel}$ is chosen for the weakly elastic fluids in this study.

(iii) The third condition relates to the capability to observe the exponential decay predicted by eq. (3) due to polymer elastic effect, or in another words, the capture frame rate (or time between two consecutive pictures $\delta t_{\mathrm{s}}$ ) needs to be sufficiently high to resolve the exponential decay regime. Rodd et al. (2005) gave an estimation of the lower detection limit for the 
relaxation time $\lambda$ accessible using the minimum resolvable diameter (Condition 2), the final aspect ratio and $\delta t_{s}$ :

$$
\lambda \geq \frac{10 \delta t_{s}}{3 \ln \left(D_{0} \Lambda_{f}^{-3 / 4} / D_{\min }\right)}
$$

where $D_{0}$ is the diameter of the pistons, $\Lambda_{\mathrm{f}}=L_{\mathrm{f}} / D_{0}$ is the final filament aspect ratio and $D_{\min }$ is the smallest diameter detectable. The prefactor 10 corresponds to the number of frames (or diameters measured) required to fit the exponential decay. In this work, the samples were stretched from an initial separation distance $L_{0}=600 \mu \mathrm{m}$ to a final distance $L_{\mathrm{f}}=1400 \mu \mathrm{m}$. This corresponded to a final filament aspect ratio of $\Lambda_{\mathrm{f}}=1.16$. With the estimated relaxation time to be measured of $\sim 100 \mu$ s and a minimum measurable diameter $D_{\min }$ of $6 \mu \mathrm{m}$ one can solve eq. (15) for the time between two consecutive pictures $\delta t_{s}=31 \mu$ s or a frame rate of $32200 \mathrm{fps}$. The final filament thinning and breaking was therefore measured at a frame rate of 45000 fps (and a decreased images size of 64x128 square pixels required to reach these high frame rates).

The ratio between the Bond number and the capillary number, defined as $\mathrm{Bo} / \mathrm{Ca}=$ $\rho g D_{0} /(2 \eta \dot{\varepsilon})$, was found to be $\sim 0.28$ ensuring that gravity does not drag the fluid below the mid-filament [Anna and McKinley (2001)]. The initial cylindrical form of the filament is ensured by an initial filament length $L_{0}$ smaller that the capillary $L_{\text {cap }}$, defined as $L_{\text {cap }}=(\sigma / \rho g)^{1 / 2}$ and estimated at $1.8 \mathrm{~mm}$ here.

\section{Filament stretching and thinning transient profiles}

The fluids of series VI have been deliberately matched in terms of viscosity (and inertia). As a consequence, the Ohnesorge number of eq. (14) will be constant for the fluids of series VI and differences observed on the experimental filament thinning transient profiles can directly be associated with the nonlinear response of the polymer chains in extensional flow. Figure 7 presents a series of images of the capillary breakup experiments using the conditions 
described in the experimental section. The overall break up time for all samples was $\sim 9.5 \mathrm{~ms}$ and longer than the piston motion time $(\sim 5.3 \mathrm{~ms})$. The high speed images in Fig. 7 show that the filament initially thins as predicted as an inviscid, inertia controlled fluid $(O h<0.2)$ with a non-uniform geometry where the filament at the top and bottom end thins faster than the midpoint. This eventually resulted in the formation of a single central droplet [Vadillo et al. (2010a)] which diameter increased with increasing polymer molecular weight (see images at $\mathrm{t}$ $=-22 \mu \mathrm{s}$ in Fig. $7 \mathrm{a}$ and $7 \mathrm{~d}$ ). The necking points above and below the droplet develop into long lasting threads with increase of molecular weight. Close examination of the last photograph of fluids with molecular weight 306 and $488 \mathrm{~kg} / \mathrm{mol}$ showed the formation of beads on the thin filament thread that eventually lead to the formation secondary droplets with diameter of order of one hundredth of the central droplet.

\section{Extensional rheology}

The extensional relaxation time is usually extracted from the mid-filament diameter $D_{\text {mid }}$ evolution using eq. (3). In the present case, an exponential thinning is observed in both filaments above and below the central droplet. As a consequence, not $D_{\text {mid }}$ but the minimum diameter $D_{\min }$ of the filaments is used in eq. (3) and (5) to extract the fluid extensional relaxation time and viscosity.

These minimum filament diameters $D_{\min }$ obtained from the high speed movies of Fig. 7 are presented in Fig. 8 as a function of time. The thinning data of the different concentration series overlap until $t \sim 1.25 \mathrm{~ms}$ or $D_{\min } \sim 60 \mu \mathrm{m}$, following an inertia controlled thinning (eq. (2), solid line in Fig. 8). After this point, which relates to a local Deborah number of $D e(t) \sim$ 1, the curves diverge, following an exponential thinning with time. The longest extensional relaxation times are obtained by fitting this exponential regime with eq. (3) (dotted lines in Fig. 8) and the data are reported in Table 2. The reproducibility of these measurements was confirmed with least three repetitive experiments and the standard deviation of the extracted $\lambda_{\text {ext }}$ is also indicated in Table 2. 
The longest relaxation time in extension obtained for PS110, PS220 and PS488 of $197 \mu$ s, 92 $\mu \mathrm{s}$ and $83 \mu$ s are the lowest reliably obtained values in uniaxial extensional flows reported so far. For all three solutions, the Deborah number (eq. (14)) is at the onset of the exponential thinning regime at $D \sim 70 \mu \mathrm{m}$ rising above unity and therefore a true elasto-capillary balance with a constant extension rate is observed. This is confirmed in Fig. 9 where a constant Weissenberg number $W i=\lambda_{\text {ext }} \dot{\varepsilon}=2 / 3$ expected for an elasto-capillary balance [Entov and Hinch (1997)] is clearly observed with a strong increase in the apparent extensional viscosity (eq. (5)). For the solution of PS306 only the onset of an elasto-capillary balance could be observed with a few data points of increasing viscosity at the lower resolution limit of the experiment close to the breaking point, indicating a possible relaxation time as low as $\sim 30 \mu \mathrm{s}$. However, for this solution an even higher optical magnification and faster recording frequency is required to improve the reliability of the observation of such short relaxation times. It should be noted that the strongly increasing apparent viscosity $\eta_{\text {e,app }}$ in Fig. 9 in the elasto-capillary balance regime represents a true transient extensional viscosity of the fluid, caused by the increasing resistance of the unravelling of the polymer chains in the uniaxial extensional flow. However, this is not the case for the apparent viscosity of the early inertiocapillary balance regime where the diameter evolution follows eq. (2) and the resistance against thinning is not related to a viscoelastic material property. Furthermore it is clear from Fig. 9 that at late times it was not possible within the resolution limits of the setup to reach the limiting theoretically predicted extensional viscosity limit of $\eta_{e} \sim 2 \lambda_{0} L^{2} c R T / M_{w}$ when approaching the finite extensibility limit of the polymer chains [Entov and Hinch (1997)].

To further increase the reliability of the extracted relaxation times, it is assured that the polymer chains have not yet reached their finite extensibility limit $L$ before or during the elasto-capillary balance. Recently, Campo-Deaño and Clasen (2010) proposed an expression of a minimum concentration, $c_{\text {low }}$, below which it is not possible to determine a relaxation time as polymer chains will be fully stretched before their elastic stresses can balance the capillary pressure: 
$c_{\text {low }}=\frac{1}{2.46} \frac{M_{w}}{N_{A} k_{B} T}\left(\frac{\sigma^{2} \rho}{\lambda^{2}}\right)^{1 / 3} L^{-3 / 2}$

Using the Zimm relaxation time $\lambda_{z}$ as an estimate for the fluid relaxation time $\lambda$, the concentration $c_{\text {low }}$ for the different polystyrene samples has been estimated (Table 2) and found to be significantly lower than the actual concentration. This also indicates that the observed exponential filament diameter decay is a true elasto-capillary balance. It should be noted that $c_{\text {low }}$ is different from the lower concentration limit $c_{\min }$ proposed in Clasen et al. (2006a). While the (lower) $c_{\min }$ indicates the critical concentration necessary to see an effect of the polymer on the thinning dynamics at all, $c_{\text {low }}$ indicates the (higher) minimal concentration above which not only an effect on the thinning but a true elasto-capillary balance is observable.

This set of reliable extensional relaxation times can now be compared to $\lambda_{0}$ from the LVE measurements. A comparison of $\lambda_{\text {ext }}$ to the LVE relaxation times in Table 2 shows that the extensional relaxation times are up to an order of magnitude larger than the LVE data. This result is consistent with the previously reported work of [Clasen et al. (2006a)] for polystyrene of much higher molecular weights between $1.810^{3} \mathrm{~kg} / \mathrm{mol}$ and $8.2710^{3} \mathrm{~kg} / \mathrm{mol}$ dissolved in DEP and in styrene oligomers. However, a direct comparison of the reduced relaxation times $\lambda_{\text {ext }} / \lambda_{z}$ shows that the assumption that $\lambda_{\text {ext }} / \lambda_{z}$ is constant for the same $c / c^{*}$ is not valid anymore. Figure 10 compares $\lambda_{\text {ext }} / \lambda_{z}$ for different molecular weights to the LVE data. For the higher molecular weights the extensional relaxation times still agree with the LVE relaxation times. However, when lowering the molecular weight the reduced extensional relaxation time starts to increase above the LVE data (up to a factor 25 for the $\mathrm{M}_{\mathrm{w}}=110.000$ $\mathrm{g} / \mathrm{mol}$ sample), even though $c / c^{*}$ is kept constant. This observation does not invalidate previously published observations that $\lambda_{\text {ext }} / \lambda_{z}$ will eventually rise above $\lambda_{0} / \lambda_{z}$ with increasing $c / c^{*}$ for a single molecular weight. However, it indicates that the onset of this increase is not solely a function of $c / c^{*}$, but also depends on the molecular weight (with the implication that the onset shifts with increasing molecular weight to higher values of $\left.c / c^{*}\right)$. 
A possible explanation for the observed influence of the molecular weight on the extensional relaxation time in Fig. 10 could be that the long-chain limit for which the power-law $[\eta] \sim M^{3 v-1}$ strictly holds is not yet reached for the molecular weights of order $10^{5} \mathrm{~g} / \mathrm{mol}$ used in series IV [Larson (2005)]. Although the experimentally determined intrinsic viscosities and their dependence on $\mathrm{M}_{\mathrm{w}}$ in Fig. 3 do not seem to show the onset of a crossover to $[\eta] \sim M^{0.5}$ at the lowest molecular weights, the polymer coil expansion $\alpha$ that can be obtained from $\alpha^{3}=\left(K / K^{\theta}\right) M^{3 v-1.5}$ (with $K^{\theta}=84 \times 10^{-3} \mathrm{ml} / \mathrm{g}$ for polystyrene at theta conditions from [Kulicke and Clasen (2004)] indicates a crossover molecular weight of $\sim 50000 \mathrm{~g} / \mathrm{mol}$, and also the solvent quality $z \sim 0.3$ (obtained from $\alpha^{3}=1.13$ for the lowest molecular weight of series VI via the Brownian dynamics simulations of [Kumar and Prakash (2003)] for $\alpha(z)$ of polystyrene) indicates the proximity to the crossover regime. The universal ratio used in eq. (12) to calculate the Zimm time will therefore be slightly overestimated for the lowest molecular weights of series IV.

However, the coil expansion is in our experiments not calculated using a (solvent quality depending) universal function [Kumar and Prakash (2003); Sunthar and Prakash (2005)], but the intrinsic viscosity (and thus $c^{*}$ ) is directly experimentally determined and used to calculate the reduced concentrations at which the material functions of different molecular weight are compared with each other. One could argue that, since the excluded volume (EV) interactions are decaying with the polymer chain approaching full stretch, the measured coil expansion at equilibrium (that includes the EV at equilibrium) are therefore not representative for the stretching chain. More specific, since the expansion and EV interactions at equilibrium depend on the solvent quality and thus on the molecular weight, and since these $M_{w}$ dependent EV interactions are getting smaller during the coil elongation, we should expect differences for the material functions that depend on the state deformation (as the relaxation time) with decreasing $M_{w}$ at the same $c / c^{*}$.

Similarly, the idea of a conformation dependent drag and therefore a dependence of the hydrodynamic interactions (HI) on the elongation of the polymer chain has been used to explain an increase of the relaxation time of the stretching chain in comparison to the coiled 
state at rest [Hsieh et al. (2003); Schroeder et al. (2004); Hsieh and Larson (2005); Sunthar and Prakash (2005); Szabo et al. (2012)]. For the case of a capillary thinning experiment Prabakhar et al. [Prabhakar et al. (2006)] have shown that including deformation dependent $\mathrm{HI}$ in their simulations predict a deviation of the critical Weissenberg number $W i=\lambda_{\text {ext }} \dot{\varepsilon}$ at the coil-stretch transition from the predicted value of 2/3 of [Entov and Hinch (1997)] due to a deviation of the critical extension rate. However, recent Brownian dynamics simulations by Somani et al. [Somani et al. (2010)] on the elongational flow of dilute polymer solutions (that take into account EV as well as conformation dependent $\mathrm{HI}$ ) indicate that not only the rate at which the coil stretch transition is observed is effected by solvent quality and $M_{w}$ but also the longest relaxation time. Moreover, their simulations indicate that the product of the two, so the Weissenberg number at which the coil-stretch transition is taking place, remains independent of changes to the molecular weight and solvent quality at $W i=2 / 3$. The relaxation times that are thus extracted from capillary breakup experiments with eq. (3) that is based on the concept of a constant Wi number should thus be independent of the changes in solvent quality originating from the varying molecular weights used in the present study, at least for the case of dilute solutions.

The question that remains is if the solutions of series IV can be considered with $c / c^{*}=0.1$ as truly dilute. The concept of a self-concentration of the expanding polymer chains in an extensional flow [Clasen et al. (2006a); Stoltz et al. (2006)] could lead even for $c / c^{*}=0.1$ to a non-dilute regime in which the molecular weight independence of the critical Weissenberg does not hold, or better, at which the reduced concentration $c / c^{*}$ at equilibrium conditions does not reflect the conditions at the onset of an expanding coil interactions. Recent Brownian simulations by Prabakhar et al. [Prabhakar and Siddarth (2011)] take into account the effect of self concentration in addition to conformation dependent $\mathrm{HI}$ and EV, however they have looked so far only at higher molecular weights closer to the long-chain limit than the $M_{w}$ used in this study. 


\section{Conclusion}

This paper investigated the viscoelastic behaviour of low viscosity polymer solutions with the aim to (a) demonstrate a reliable determination of very short relaxation times in uniaxial extension and in small amplitude oscillations, and (b) use this reliable data to compare the relaxation times in shear and extension for different molecular weights at the same overlap concentration $c / c^{*}$. Relaxation times in the LVE limit were obtained with high frequency SAOS experiments using the Piezo Axial Vibrator (PAV) whereas extensional data have been obtained from filament thinning experiments using the Cambridge "Trimaster" and high speed visualisation. Concentration series of mono disperse low molecular weight polystyrenes dissolved in DEP have been characterised within the terminal relaxation regime to obtain intrinsic viscosities and critical concentrations $c^{*}$. The longest shear relaxation times have been obtained from fitting the LVE data with the Zimm relaxation spectra and it was possible to obtain shear relaxation times down to values of $\sim 10 \mu \mathrm{s}$. With this data set it was reconfirmed that, within the LVE limit, also for weakly elastic fluids the reduced longest relaxation time $\lambda_{0} / \lambda_{z}$ is solely a function of the overlap parameter $c / c^{*}$.

The longest relaxation times in extensional flow $\lambda_{\text {ext }}$ were determined from capillary thinning experiments for a series of solutions with varying molecular weight but a constant overlap parameter $c / c^{*} \sim 0.1$ (and therefore matched shear viscosity of $\sim 12 \mathrm{mPa} \mathrm{s}$ at $25^{\circ} \mathrm{C}$ ). For these solutions extensional relaxation times could reliably be obtained down to values of $\sim 80 \mu \mathrm{s}$ which represent the lowest so far measured relaxation times in extension determined from capillary thinning experiments. It could be shown that, different from the LVE relaxation times, the reduced relaxation times $\lambda_{\text {ext }} / \lambda_{\mathrm{z}}$ are not constant for a single overlap parameter $c / c^{*}$, but also depend on the molecular weight. Comparing $\lambda_{\text {ext }}$ with $\lambda_{0}$ at a constant $\mathrm{c} / \mathrm{c}^{*}$ shows that $\lambda_{\text {ext }} / \lambda_{0}$ is increasing with decreasing $M_{\mathrm{w}}$.

This experimental capability of measuring very short relaxation time can bring new insight in the understanding of the fluid microstructure of low viscosity polymer solutions as well as providing experimental data to an area of rheology limited, until now, to simulations. 


\section{Acknowledgment}

CC and WM acknowledge financial support from the ERC starting grant no. 203043NANOFIB. DV acknowledges financial support from the Engineering and Physical Sciences Research Council (UK) and industrial partners in the Innovation in Industrial Inkjet Technology project, EP/H018913/1 as well as Dr T. Tuladhar and Dr S. Hoath for discussions. The authors acknowledge Prof. M. Mackley for fruitful discussions and Dr S. Butler for his help in low viscosity measurements.

\section{References}

Anna S.L. and G.H. Mckinley, "Elasto-capillary thinning and breakup of model elastic liquids", J. Rheol., 45, 115-138 (2001).

Anna S.L., G. McKinley, D.A. Nguyen, T. Sridhar, S.J. Muller, J. Huang, D.F. James, “An interlaboratory comparison of measurements from filament-stretching rheometers using common test fluids", J. Rheol., 45, 83-114 (2001).

Arnolds O., H. Buggisch, D. Sachsenheimer and N. Willenbacher, "Capillary breakup extensional rheometry (CaBER) on semi-dilute and concentrated polyethyleneoxide (PEO) solutions", Rheol. Acta, 49, 1207-1217 (2010).

Bach A., H. Koblitz Rasmussen and O. Hassager, "Extensional viscosity for polymer melts measured in the filament stretching rheometer”, J. Rheol., 47 (2), 429-441 (2003).

Bazilevsky A. V., V. M. Entov and A.N. Rozhkov, "Liquid filament microrheometer and some of its applications", Third European Rheol. Conf., (Ed. D.R. Oliver) Elsevier Applied Science, 41-43 (1990). 
Bazilevsky A.V., V.M. Entov and A.N. Rozhkov, "Failure of polymer solutions filaments", Pol. Sc. Series B, 39, 316-324 (1997).

Bazilevsky A.V., V.M. Entov, A.N. Rozhkov, "Breakup of an Oldroyd liquid bridge as a method for testing the rheological properties of polymer solutions”, Polymer Science, 43, 716 (2001).

Brenner M.P., J.R. Lister and H.A. Stone, "Pinching threads, singularities and the number 0.0304”, Phys. Fluids, 8, 2827-2836 (1996).

Campo-Deaño L. and C. Clasen, "The slow retraction method (SRM) for the determination of ultra-sort relaxation times in capillary breakup extensional rheometry experiments", J. NonNewtonian Fluid Mech., 165, 1688-1699 (2010).

Clasen C., J.P. Plog, W.-M Kulicke, M. Owens, C. Macosko, L.E. Scriven, M. Verani and G.H. McKinley, "How dilute are dilute solutions in extensional flows?", J. Rheol., 50, 849$881(2006 a)$.

Clasen C., J. Eggers, M.A. Fontelos, J. Li, G.H. McKinley, "The beads-on-string structure of viscoelastic threads", Journal of Fluid Mechanics, 556, 283-308 (2006b).

Clasen C., J. Bico, V. Entov, G.H. McKinley, “'Gobbling drops': the jetting-dripping transition in flows of polymer solutions", Journal of Fluid Mechanics, 636, 5-40 (2009).

Clasen C., "Capillary breakup extensional rheometry of semi-dilute polymer solutions", Korea-Aust. Rheol. J., 22(4), 331-338 (2010).

Clasen C., P.M. Phillips, Lj. Palangetic, J. Vermant, “Dispensing of Rheologically Complex Fluids: the Map of Misery”, AIChE Journal (2012), DOI: 10.1002/aic.13704. 
Crassous J., R. Régisser, M. Ballauff and N. Willenbacher, "Characterisation of the viscoelastic behaviour of complex fluids using the piezoelastic axial vibrator", J. Rheol., 49, $851-863$ (2005).

Day R. F., E. J. Hinch, and J. R. Lister, "Self-similar capillary pinchoff of an inviscid fluid", Phy. Rev. Let., 80(4), 704-707 (1998).

Eggers J., "Nonlinear dynamics and breakup of free-surface flows", Rev. Mod. Phys., 69, 865-929 (1997).

Entov V.M., A.L. Yarin, Influence of elastic stresses on the capillary breakup of jets of dilute polymer solutions, Fluid Dynamics, 19, 21-29 (1984).

Entov V.M. and E.J. Hinch, "Effect of a spectrum relaxation times on the capillary thinning of a filament elastic liquids", J. Non-Newtonian Fluid Mech., 72, 31-53 (1997).

Erni P., M. Varagnat, C. Clasen, J. Crest, G.H. McKinley, "Microrheometry of Sub-Nanoliter Biopolymer Samples: Non-Newtonian Flow Phenomena of Carnivorous Plant Mucilage”, Soft Matter, 7, 10889 (2011).

Groß T., L. Kirschenmann and W. Pechhold, "Piezo Axial Vivrator (PAV) - A new oscillating squeeze flow rheometer", Proceedings Eurheo, (Ed. H. Munsted, J. Kaschta, A. Merten) Erlangen (2002).

Gupta R.K., D.A. Nguyen and T. Sridhar, "Extensional viscosity of dilute polystyrene solutions: effect of concentration and molecular weight”, Phys. Fluids, 13(6), 1296-1317 (2000).

Graessley, W. W., "Polymer chain dimensions and the dependence of viscoelastic properties on the concentration, molecular weight and solvent power," Polymer, 21, 258-262 (1980). 
Harrison G. M., J. Remmelgas, and L. G. Leal, "The dynamics of ultradilute polymer solutions in transient flow: Comparison of dumbbell-based theory and experiment," J. Rheol., 42, 1039-1058 (1998).

Hoath S.D., I.M. Hutchings, G.D. Martin, T.R. Tuladhar, M.R. Mackley, and D.C Vadillo, "Link between ink rheology, drop-on-demand jet formation and printability“, J. Imaging Sci. Tech., 53(4), 041208 (2009).

Hsieh, C. C. and R. G. Larson, "Prediction of coil-stretch hysteresis for dilute polystyrene molecules in extensional flow". Journal of Rheology, 49, 1081-1089 (2005).

Hsieh, C. C., L. Li and R. G. Larson, "Modeling hydrodynamic interaction in Brownian dynamics: simulations of extensional flows of dilute solutions of DNA and polystyrene". Journal of Non-Newtonian Fluid Mechanics, 113, 147-191 (2003).

Huertas A. and G. Medioni, Detection of intensity changes with subpixel accuracy using Laplacian-Gaussian Masks, IEEE Trans. Pattern Anal. Mach. Intell., vol. Pami-8 (5), 651-664 (1986).

Kirschenmann L., PhD thesis, Institut für dynamische Materialprüfung (IdM), University of $\operatorname{Ulm}(2003)$.

Kojic N., J. Bico, C. Clasen, G.H. McKinley, „Ex vivo rheology of spider silk”, The Journal of Experimental Biology, 209, 4355-4362 (2006).

Kolte M.I. and P. Szabo, “Capillary thinning of polymeric filaments”, J. Rheol., 43, 609-625 (1999).

Kulicke, W.-M. and C. Clasen, Viscosimetry of Polymers and Polyelectrolytes, Springer, Heidelberg (2004). 
Kumar, K. S. and J. R. Prakash, "Equilibrium swelling and universal ratios in dilute polymer solutions: Exact Brownian dynamics simulations for a delta function excluded volume potential", Macromolecules, 36, 7842-7856 (2003).

Larson, R. G., "The rheology of dilute solutions of flexible polymers: Progress and problems". Journal of Rheology, 49, 1-70 (2005).

Liang R.F. and M.R. Mackley, "Rheological characterisation of the time and strain dependence for polyisobutylene solutions", J. Non-Newtonian Fluid Mech., 52, 387-405 (1994).

Ma W.K.A., F. Chinesta, T. Tuladhar and M.R. Mackley, "Filament stretching of carbon nano tube suspension", Rheol. Acta, 47, 447-457 (2008).

Marr D. and E. Hildreth, "Theory of edge detection", Proc. R. Soc. Lond B, 207, 187-217 (1980).

McKinley G.H., "Visco-Elasto-Capillary thinning and break-up of complex fluid”, Rheology Reviews 2005, The British Soc. Rheol., 1-49 (2005).

McKinley G.H. and A. Tripathi, "How to extract the Newtonian viscosity from a capillary break up measurement in a filament rheometer", J. Rheol, 44, 653-670 (2000).

McKinley G.H and G.H. and Sridhar T., "Filament stretching rheometry of complex fluids", Annual Rev. Fluids Mech., 34, 375-415 (2002).

Miller E., C. Clasen, J.P. Rothstein, "The effect of step-stretch parameters on capillary breakup extensional rheology (CaBER) measurements”, Rheol. Acta, 48, 625-639 (2009).

Nguyen, T.Q. and H. H. Kausch, "Flexible Polymer Chains in Elongational Flow: Theory \& Experiment“", Springer-Verlag, Berlin, (1999).

Öttinger, H. C., “Stochastic Processes in Polymeric Liquids”, Springer Verlag, Berlin, (1996). 
Plog J.P., W.M. Kulicke, C. Clasen, "Influence of the molar mass distribution on the elongational behaviour of polymer solutions in capillary breakup", Appl. Rheol., 15, 28-37 (2005).

Prabhakar, R., J. R. Prakash and T. Sridhar, "Effect of configuration-dependent intramolecular hydrodynamic interaction on elasto-capillary thinning and break-up filaments of dilute polymer solutions". Journal of Rheology, 50, 925-947(2006).

Prabhakar, R. and G. Siddarth, "The role of coil-stretch hysteresis in the capillary breakup of dilute polymer solutions." The Society of Rheology 83rd Annual Meeting. Cleveland, Ohio (USA), (2011).

Regev O., S. Vandebril, E. Zussman, C. Clasen, "The role of interfacial viscoelasticity in the stabilization of an electrospun jet”, Polymer, 51, 2611-2620 (2010).

Renardy M., "Some comments on the surface tension driven breakup (or the lack of it) of the viscoelastic jets", J. Non-Newtonian Fluid Mech., 51, 97-107 (1994).

Renardy M., "A numerical study of the asymptotic evolution and breakup of Newtonian and viscoelastic jets”, J. Non-Newtonian Fluid Mech., 59, 267-282 (1995).

Rodd, L.E., T.P. Scott, J.J. Cooper-White and G.H. McKinley, "Capillary Breakup Rheometry of Low-Viscosity Elastic Fluids”, Appl. Rheol., 15(1), 12-27, (2005).

Sattler, R., C. Wagner, J. Eggers, "Blistering pattern and formation of nano fibers in capillary thinning of polymer solutions" Physical Review Letters, 100, 164502 (2008).

Schroeder, C. M., E. S. G. Shaqfeh and S. Chu, "Effect of hydrodynamic interactions on DNA dynamics in extensional flow: Simulation and single molecule experiment", Macromolecules, 37, 9242-9256 (2004). 
Sharma, V., A.M. Ardekani and G.H. McKinley, "Beads on a String' Structures and Extensional Rheometry using Jet Break-up", 5th Pacific Rim Conference on Rheology (PRCR-5), (2010).

Somani, S., E. S. G. Shaqfeh and J. R. Prakash, "Effect of Solvent Quality on the Coil-Stretch Transition", Macromolecules, 43, 10679-10691 (2010).

Spiegelberg S. H. and G.H. McKinley, "Stress relaxation and elastic decohesion of viscoelastic polymer solutions in extensional flow", J. Non-Newtonian Fluid Mech., 67, 49-76 (1996).

Spiegelberg S.H., D.C. Ables, G.H. McKinley, "The role of end-effects on measurements of extensional viscosity in filament stretching rheometers", J. Non-Newtonian Fluid Mech, 64 229-267 (1996).

Sridhar T., "An overview of the project M1", J. Non-Newtonian Fluid Mech., 35, 85-92 (1990).

Stelter M., G. Brenn, A.L. Yarin, R.P. Singh, F. Durst, "Investigation of the elongation behavior of polymer solutions by means of an elongational rheometer", J. Rheol., 46, 507-527 (2002).

Stoltz, C., J.J. De Pablo and M.D. Graham, "Concentration dependence of shear and extensional rheology of polymer solutions: Brownian dynamics simulations", Journal of Rheology, 50, 137-167 (2006).

Sunthar, P. and J. R. Prakash, "Parameter-free prediction of DNA conformations in elongational flow by successive fine graining", Macromolecules, 38, 617-640 (2005).

Szabo, P., G.H. McKinley and C. Clasen, "Constant force extensional rheometry of polymer solutions", J. Non-Newtonian Fluid Mech., 169-170, 26-41 (2012). 
Tirtaatmadja V., G.H. McKinley, J.J. Cooper-White, Drop formation and breakup of low viscosity elastic fluids: effects of molecular weight and concentration, Phys. Fluids, 18, $043101(2006)$.

Tuladhar T.R. and M.R. Mackley, "Filament stretching rheometry and break-up behaviour of low viscosity polymer solutions and ink jets fluids", J. Non-Newtonian Fluid Mech., 148, $97-$ $108(2008)$.

Vadillo D.C., T.R. Tuladhar, A.C. Mulji, M.R. Mackley, S. Jung and S.D. Hoath, "The development of the "Cambridge Trimaster" filament stretch and break-up device for the evaluation of ink jet fluids", J. Rheol., 54(2), 261-282 (2010a).

Vadillo D.C., T. R. Tuladhar, A. Mulji, M.R. Mackley, "The Rheological characterisation of linear viscoelasticity for ink jet fluids using a Piezo Axial Vibrator (PAV) and Torsion Resonator (TR) rheometers", J. Rheol, 54(4), 781-79 (2010b).

Vananroye A., P. Leen, P. Van Puyvelde, C. Clasen, "TTS in LAOS: validation of timetemperature superposition under large amplitude oscillatory flow”, Rheol. Acta, 50, 795-807 (2011)..

Yesilata B., C. Clasen, G.H. McKinley, "Nonlinear Shear and Extensional Flow Dynamics of wormlike surfactant solutions”, J. Non-Newtonian Fluid Mech., 133, 73-90 (2006). 
a)

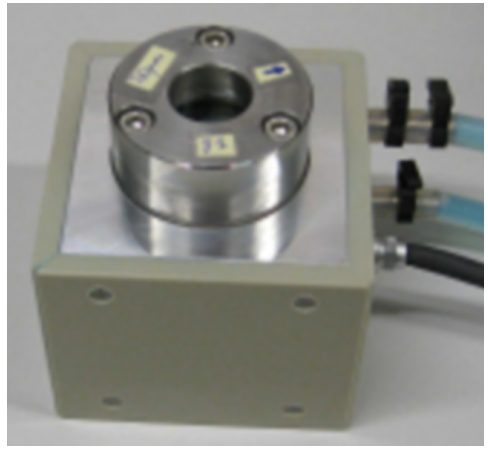

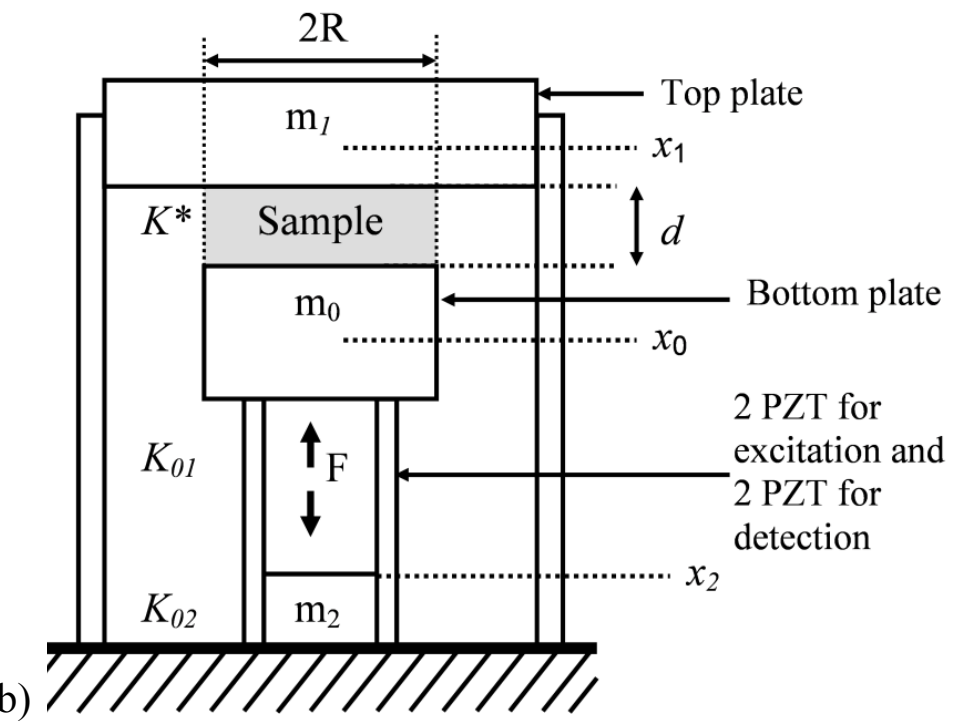

Figure 1: (a) Image and (b) schematic drawing of the PAV (reproduced from Kirschenmann (2003) and Crassous et al. (2005)). 


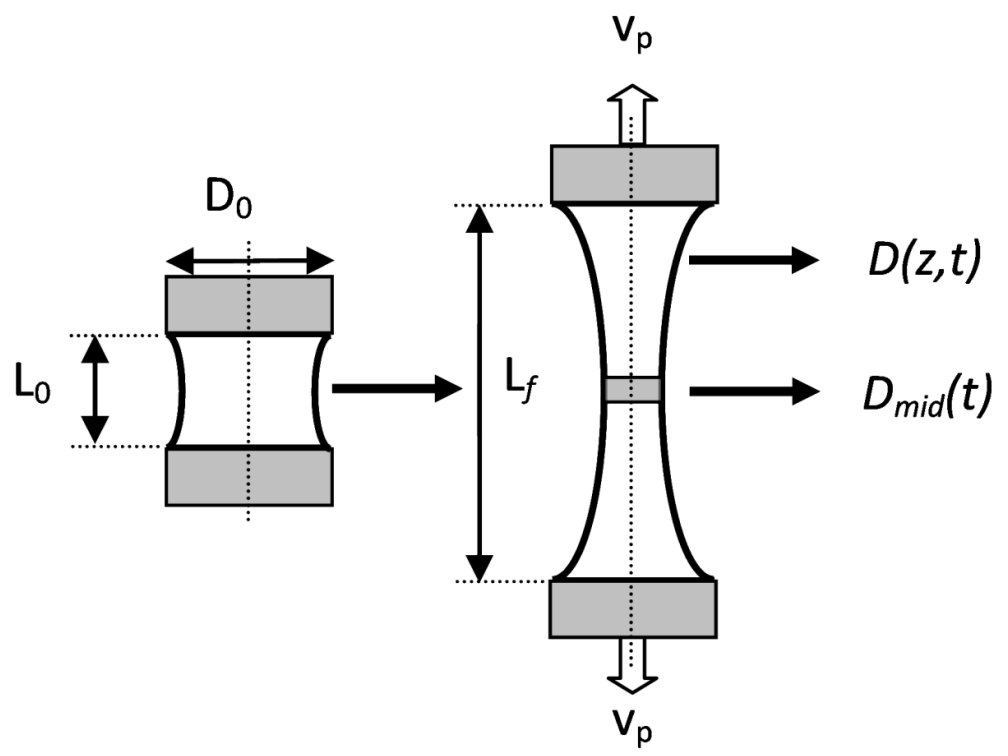

Figure 2: Principle of a capillary thinning experiment. 


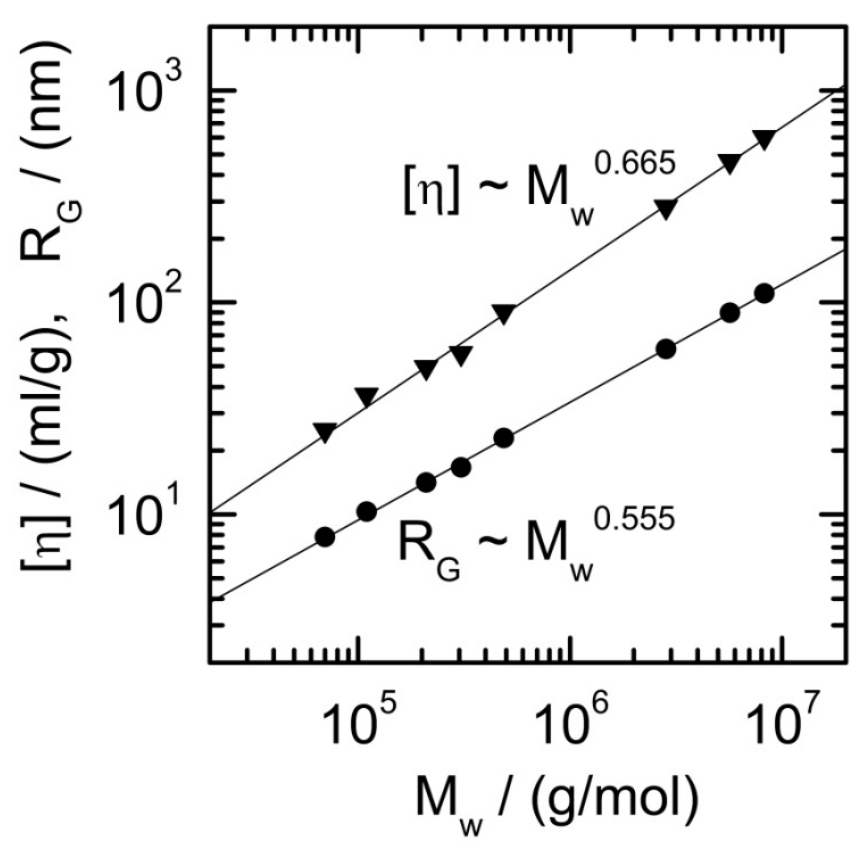

Figure 3: Intrinsic viscosity $[\eta]$ and gyration radius $R_{\mathrm{g}}$ as a function of molecular weight for polystyrene in diethyl phthalate. Data for $M_{\mathrm{w}}>10^{6} \mathrm{~g} / \mathrm{mol}$ are taken from Clasen et al. (2006a). 


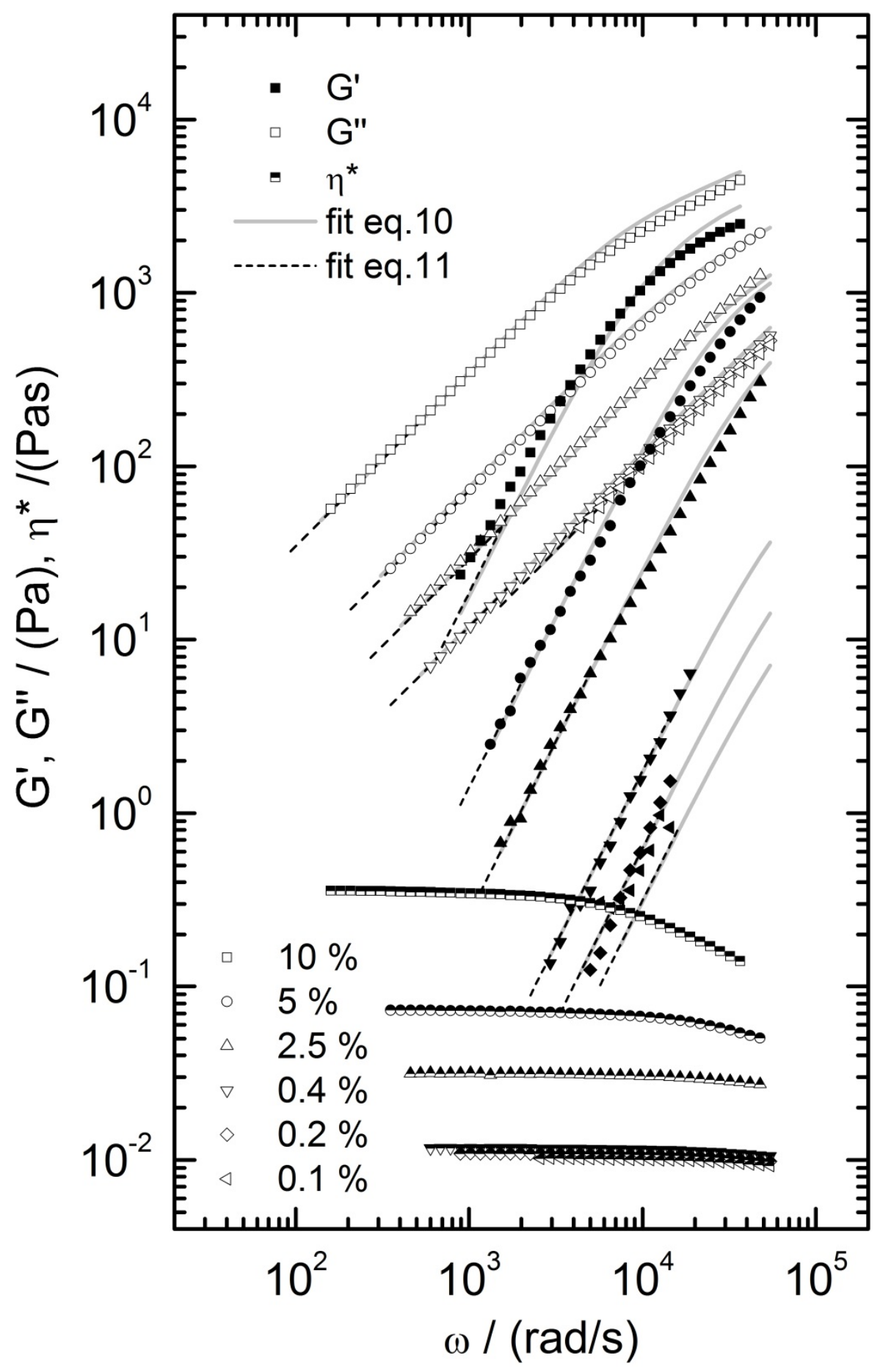

Figure 4: Loss modulus $G^{\prime \prime}$, storage modulus $G^{\prime}$ and complex viscosity $\eta^{*}$ as a function of the frequency for the dilution series II (PS with $\mathrm{M}_{\mathrm{w}}=110000 \mathrm{~g} / \mathrm{mol}$ in DEP). The solid lines represent fits of the Zimm spectrum of eq. (10) to the $G^{\prime}$ and $G$ ', data, the dashed lines are fits of solely the terminal relaxation regime using eq. (11) in order to extract the longest relaxation time as the only fitting parameter. 


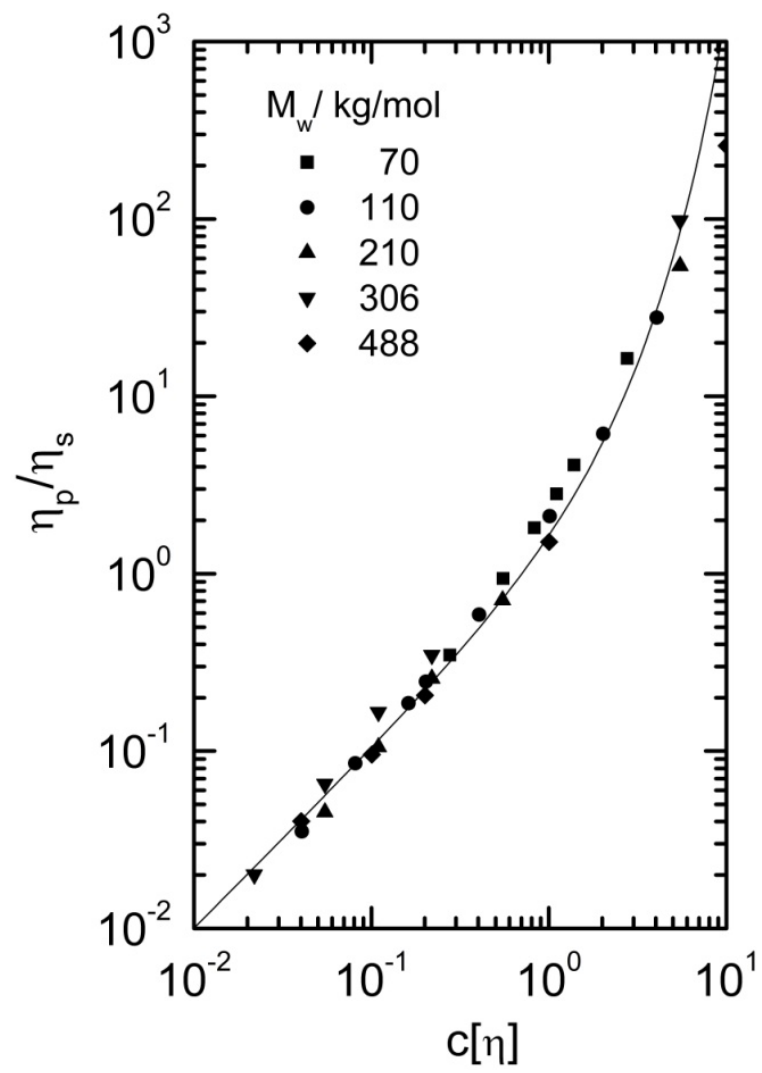

a)

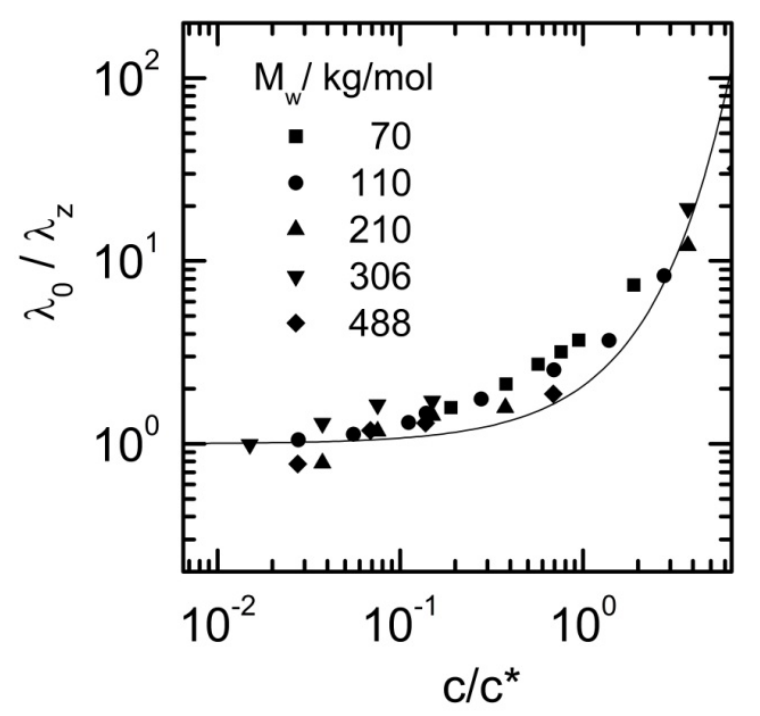

b)

Figure 5: (a) Specific viscosity $\eta_{\mathrm{p}} / \eta_{\mathrm{s}}$ as a function of the overlap parameter $c[\eta]$ for PS in DEP for the dilution series I-V. The solid line is a fit of the Martin equation $\eta_{p}=\eta_{s} c[\eta] e^{K_{M} c[\eta]}$.

Reduced relaxation time $\lambda_{0, \mathrm{fit}} / \lambda_{z}$ (obtained from fitting the LVE data of the PAV experiments 
with eq. (11)) as a function of the reduced concentration $c / c^{*}$ for the dilution series I-VI. The solid line is the theoretical prediction of eq. (13). 


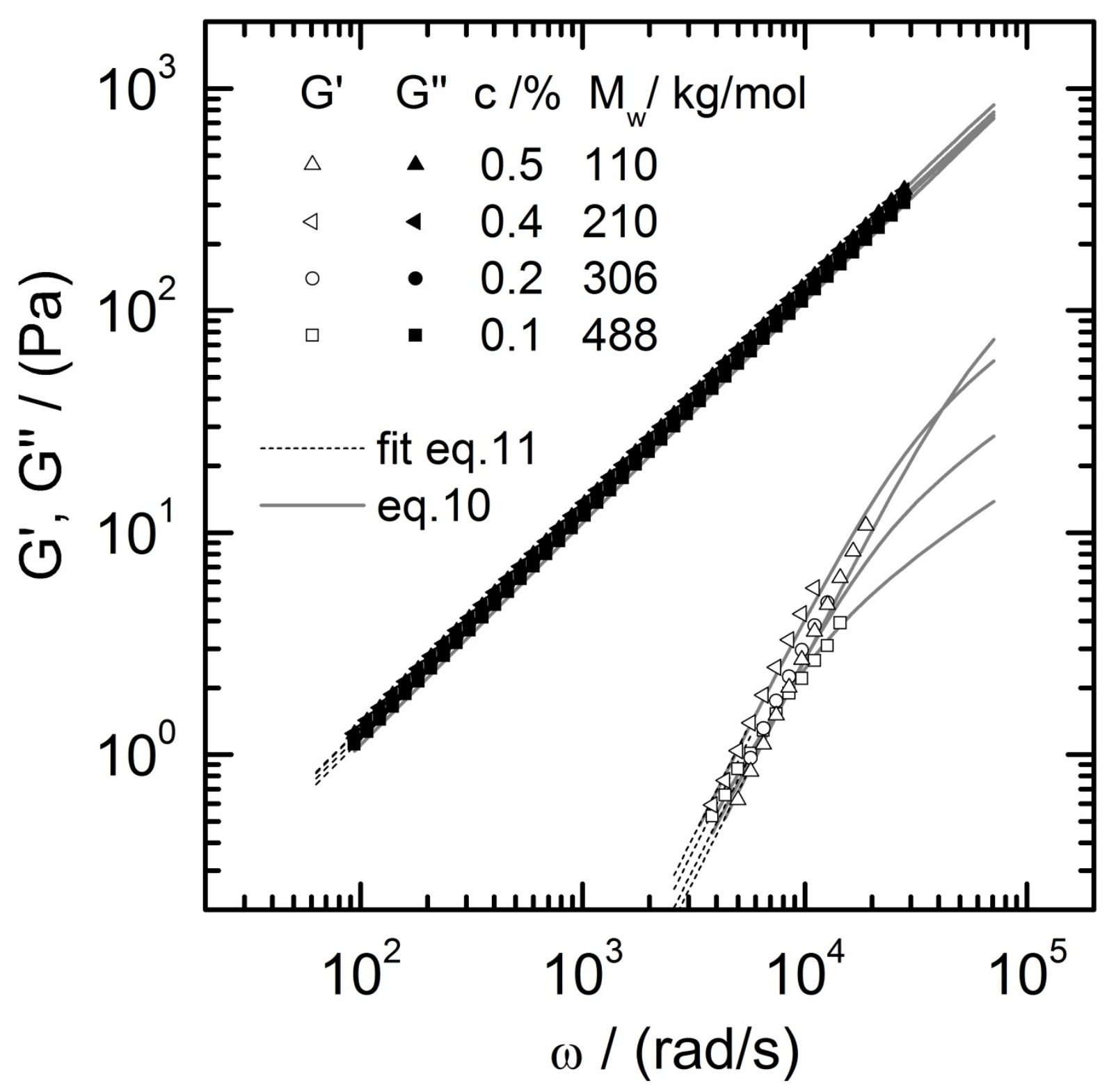

Figure 6: Loss modulus $G$ ' and storage modulus $G^{\prime}$ as a function of the frequency for the dilution series VI (PS in DEP with different molecular weights and concentrations, but similar reduced concentration of $c / c^{*} \sim 0.1$ and similar zero-shear viscosity of $\eta_{0} \sim 12 \mathrm{mPa}$ ). The solid lines represent fits of the Zimm spectrum of eq. (10) to the $G$ ' and $G$ ', data, the dashed lines are fits of solely the terminal relaxation regime using eq. (11) in order to extract the longest relaxation time as the only fitting parameter. 
a
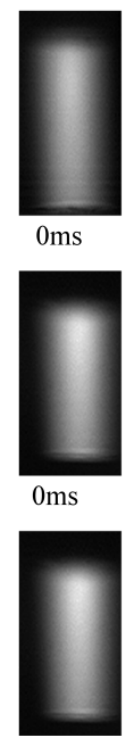

$0 \mathrm{~ms}$

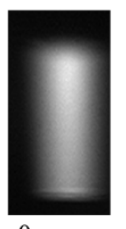

0ms

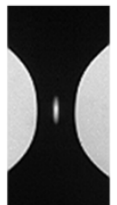

$-0.131$

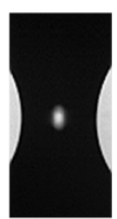

$-0.131$

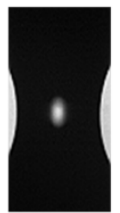

$-0.131$

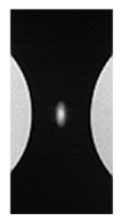

$-0.131$
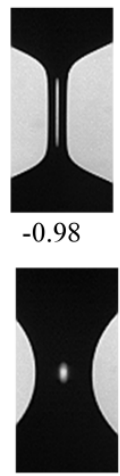

$-0.98$

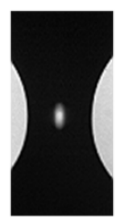

$-0.98$

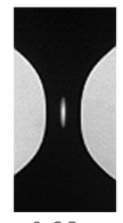

$-0.98$

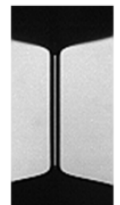

$-0.64$

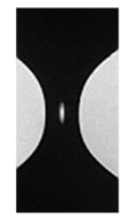

$-0.64$

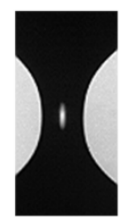

$-0.64$

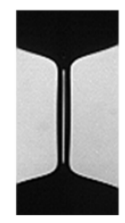

$-0.64$

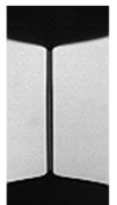

$-0.31$

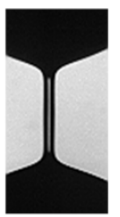

$-0.31$
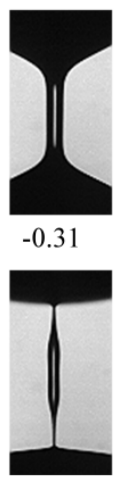

$-0.31$

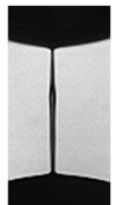

$-0.089$

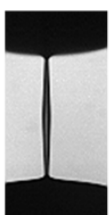

$-0.089$

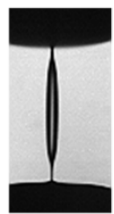

$-0.089$

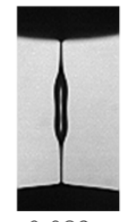

$-0.089$

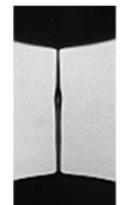

$-0.067$

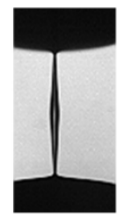

$-0.067$

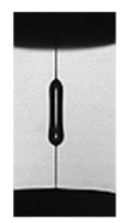

$-0.067$

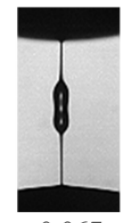

$-0.067$
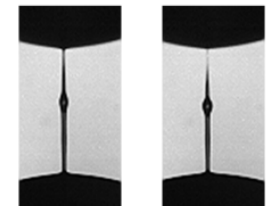

$-0.044$

$-0.022$
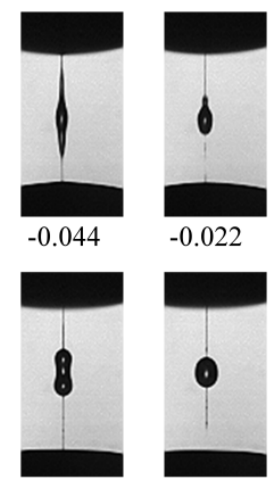

$-0.022$
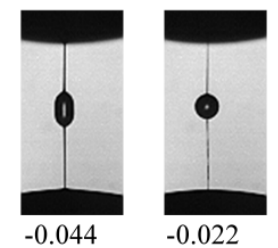

$-0.044 \quad-0.022$
Break-up
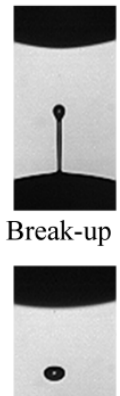

Break-up
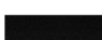

$\odot$

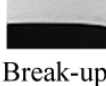

(areak

-

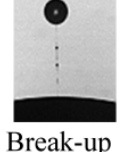

Figure 7: Images from the last $1.3 \mathrm{~ms}$ of the capillary break up of the dilution series VI of PS in DEP: (a) PS110 at $0.5 \mathrm{wt} \%$, (b) PS210 at $0.4 \mathrm{wt} \%$, (c) PS306 at $0.2 \mathrm{wt} \%$, (d) PS488 at $0.1 \mathrm{wt}$. Images are taken from high speed movies recorded at $45000 \mathrm{fps}$ with an exposure time of 3 $\mu \mathrm{s}$. The break up time is used as time reference. 


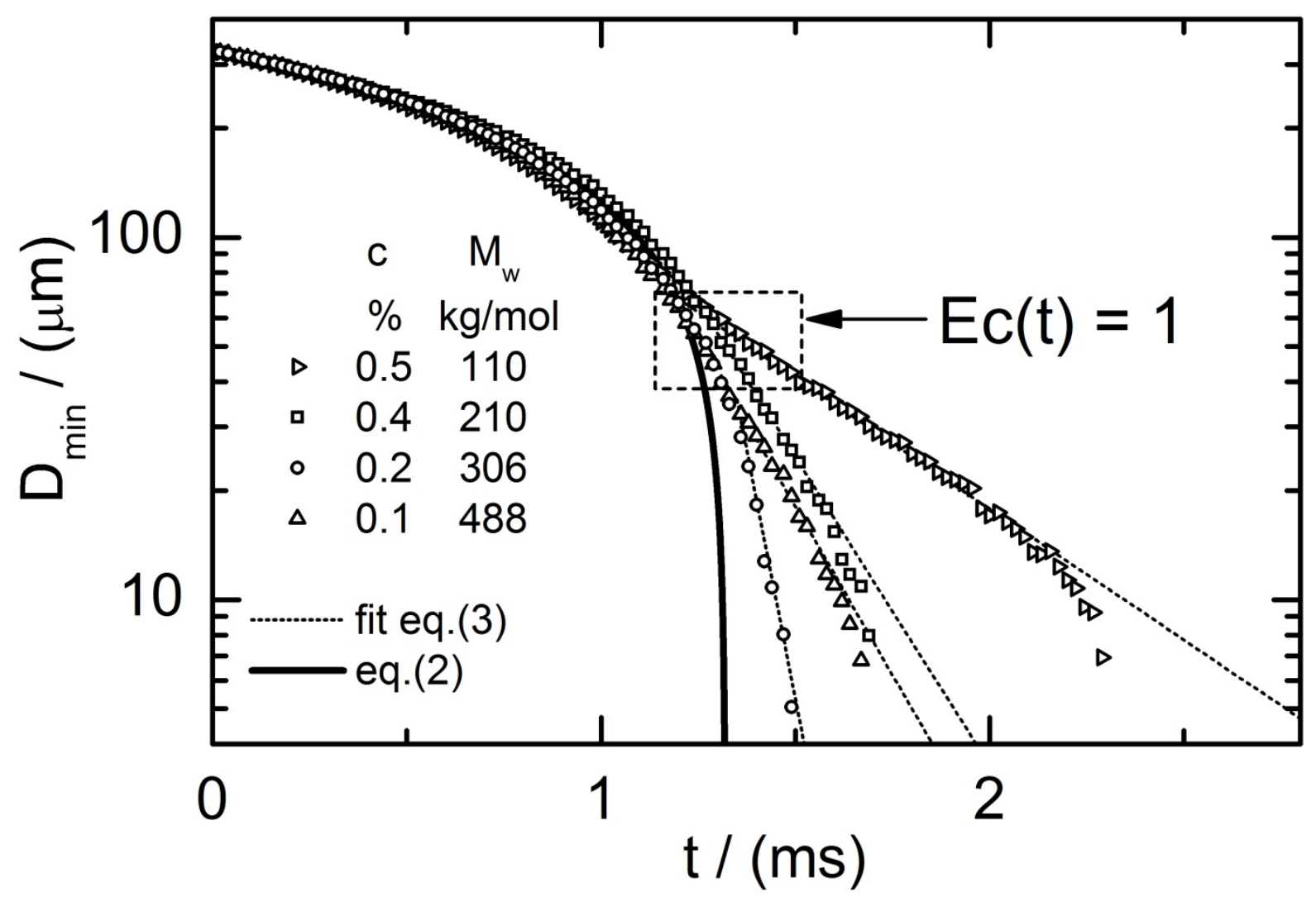

Figure 8: Evolution of the minimum filament diameter $D_{\min }$, obtained from the processed images of the high speed movies of Fig. 7, as a function of time. The solid line represents a purely inertia controlled thinning following eq. (2). Dotted lines represent fits of the elastocapillary balance regime with eq. (3). 


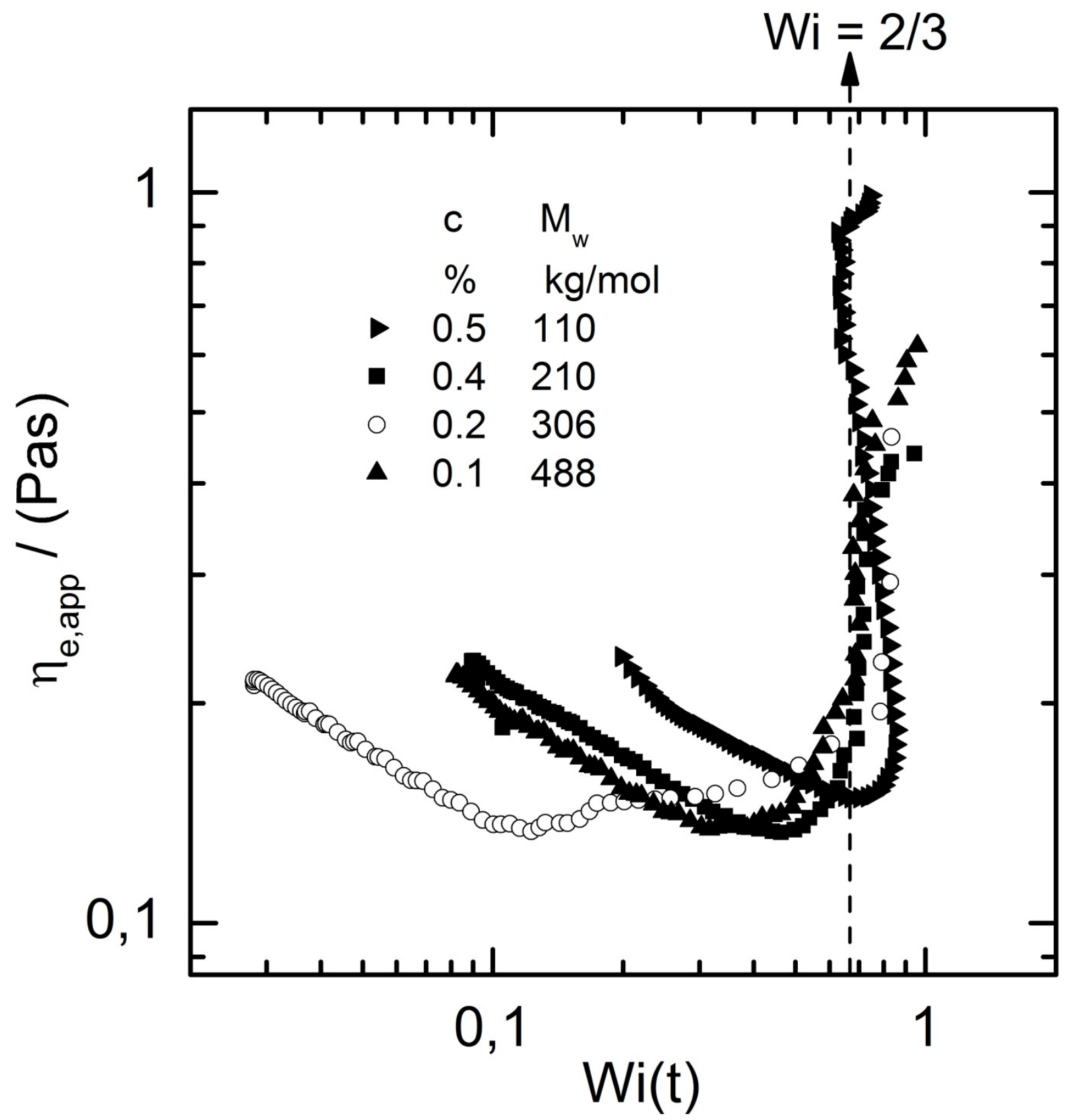

Figure 9: Apparent extensional viscosity $\eta_{\mathrm{e}, \text { app }}$ as a function of the Weissenberg number $W i=$ $\lambda_{\text {ext }} \dot{\varepsilon}$ calculated with eqs. (5) and (4) from the diameter data of Fig. 8. The dashed line represents the natural Weissenberg number for the filament thinning in the elasto-capillary balance regime of $W i=2 / 3$. 


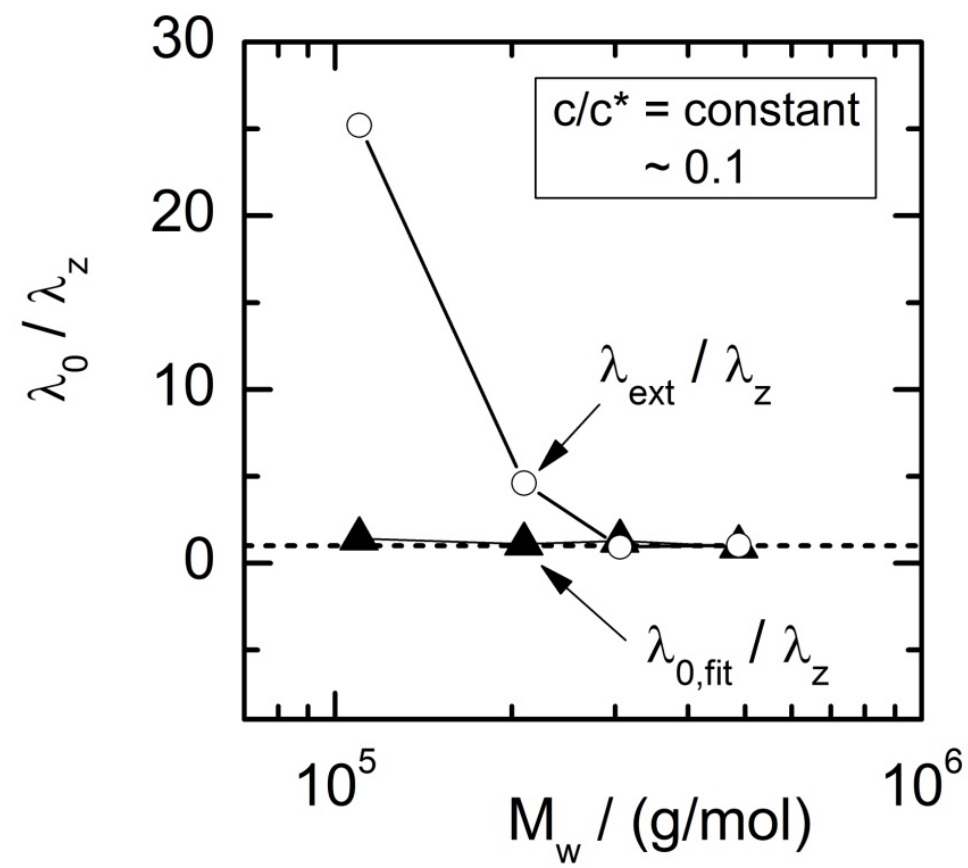

Figure 10: Comparison of the reduced relaxation times in LVE shear and uniaxial extension as a function of the molecular weight at a constant reduced concentration $c / c^{*}$. 